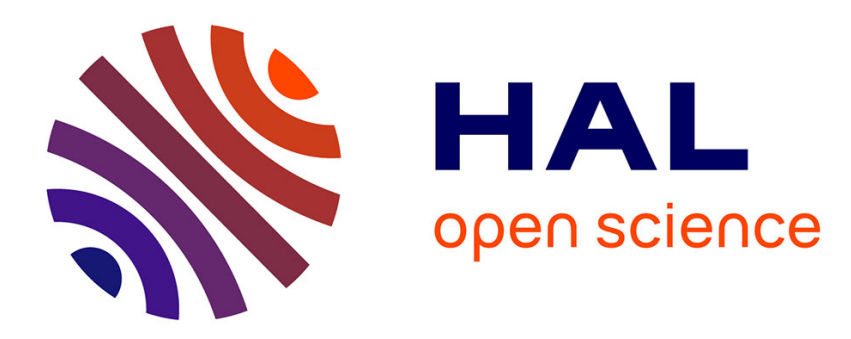

\title{
Modeling Fractures and Barriers as Interfaces for Flow in Porous Media
}

Jérôme Jaffré, Vincent Martin, Jean Roberts

\section{To cite this version:}

Jérôme Jaffré, Vincent Martin, Jean Roberts. Modeling Fractures and Barriers as Interfaces for Flow in Porous Media. [Research Report] RR-4848, INRIA. 2003. inria-00071735

\section{HAL Id: inria-00071735 \\ https://hal.inria.fr/inria-00071735}

Submitted on 23 May 2006

HAL is a multi-disciplinary open access archive for the deposit and dissemination of scientific research documents, whether they are published or not. The documents may come from teaching and research institutions in France or abroad, or from public or private research centers.
L'archive ouverte pluridisciplinaire HAL, est destinée au dépôt et à la diffusion de documents scientifiques de niveau recherche, publiés ou non, émanant des établissements d'enseignement et de recherche français ou étrangers, des laboratoires publics ou privés. 


\title{
Modeling Fractures and Barriers as Interfaces for Flow in Porous Media
}

\author{
Jérôme Jaffré — Vincent Martin — Jean E. Roberts
}

$\mathbf{N}^{\circ} 4848$

June 2003

THÈME 4 



\title{
Modeling Fractures and Barriers as Interfaces for Flow in Porous Media
}

\author{
Jérôme Jaffré $^{*}$, Vincent Martin ${ }^{\dagger}$, Jean E. Roberts ${ }^{\ddagger}$ \\ Thème 4 - Simulation et optimisation \\ de systèmes complexes \\ Projets Estime \\ Rapport de recherche $\mathrm{n}^{\circ} 4848$ - June 2003 - 27 pages
}

\begin{abstract}
We consider a fractured porous medium that is studied at a scale such that the fractures can be modeled individually. Models for flow in which the fractures are interfaces between subdomains are presented. These models take into account interactions between the fractures and the surrounding porous medium. Existence and uniqueness of the solution to the model problem is proved. Error estimates show convergence as $O(\max (h, d))$ in the L2 norm, where $h$ is the mesh size and $d$ the fracture width. Numerical experiments confirm the theorical results.
\end{abstract}

Key-words: fractures, faults, barriers, mixed finite elements, flow in porous media, domain decomposition

\footnotetext{
* Inria Rocquencourt, BP 105, 78153 Le Chesnay Cedex, France, Jerome.Jaffre@inria.fr

$\dagger$ Inria Rocquencourt and Andra, Parc de la Croix Blanche, 1-7 rue Jean Monnet, 92298 Chatenay-Malabry, Vincent. Martin@inria.fr

$\ddagger$ Inria Rocquencourt, BP 105, 78153 Le Chesnay Cedex, France, Jean.Roberts@inria.fr
} 


\section{Modélisation de fractures et de barrières comme des interfaces pour la simulation de l'écoulement en milieu poreux}

Résumé : Nous nous intéressons à l'écoulement dans un milieu poreux fracturé qui est étudié à une échelle telle que les fractures peuvent être modélisées individuellement. Nous présentons des modèles d'écoulement dans lesquels les fractures sont représentées comme des interfaces entre sous-domaines. Ces modèles prennent en compte les intéractions entre les fractures et le milieu poreux environnant. L'existence et l'unicité de la solution du problème modèle sont prouvées; des estimations d'erreurs montrent une convergence en $O(\max (h, d))$ pour la norme L2, où $h$ est le pas du maillage et $d$ l'épaisseur de la fracture. Des expériences numériques confirment les résultats théoriques.

Mots-clés : fractures, failles, barrières, éléments finis mixtes, écoulement en milieu poreux, décomposition de domaines 


\section{Table of Contents}

1 Introduction $\quad 4$

2 Description of the problem $\quad 4$

3 Derivation of the model $\quad 5$

3.1 Averaging across the fracture . . . . . . . . . . . . . . . 6

3.1.1 Averaging the conservation equation . . . . . . . . . . . . 6

3.1 .2 Averaging Darcy's law . . . . . . . . . . . . . . . . . . 6

4 Model problem for the fracture $\quad 8$

4.1 Strong formulation of the model problem . . . . . . . . . . . . . . . 8

4.2 Weak formulation of the model problem . . . . . . . . . . . . . . 9

4.3 Existence and uniqueness of the solution . . . . . . . . . . . . . . . . 10

5 Interpretation of the discrete model and error estimate 12

5.1 Assumptions concerning the mesh . . . . . . . . . . . . . . . . 12

5.2 Discrete transmission multiblock problem . . . . . . . . . . . . . . . 12

5.3 Discrete model problem . . . . . . . . . . . . . . . . . . . . . . 13

5.4 Link between transmission and model problems . . . . . . . . . . . . . . . . . 14

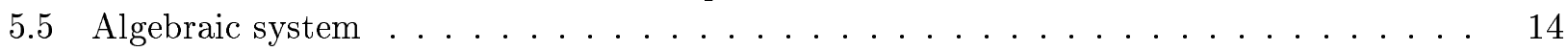

5.6 Error estimates . . . . . . . . . . . . . . . . . . . . . . 15

6 Numerical results $\quad 16$

6.1 First test-case : large permeability in the fracture and Dirichlet boundary conditions . 17

6.2 Second test-case : small permeability in the fracture and Neumann boundary conditions 18

6.3 Third test-case : 2 anisotropic permeabilities in the fracture and Dirichlet boundary

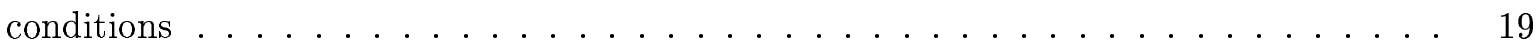

7 Domain decomposition $\quad \mathbf{2 1}$

7.1 Domain decomposition formulation . . . . . . . . . . . . . . . 21

7.2 Weak formulation . . . . . . . . . . . . . . . . . . . . . . . . . . . . . . . . . . . . .

7.3 Solving the system efficiently . . . . . . . . . . . . . . . . 24

8 Conclusion $\quad 24$ 


\section{Introduction}

We are concerned with flow in a fractured porous medium. Our study is carried out at a scale for which the fractures can be modeled individually. The fractures have a small width and are treated as interfaces between subdomains. We also assume that the fractures are filled with debris and that flow in the fractures respects Darcy's law.

We distinguish two types of fractures: fractures which have a permeability higher than that in the surrounding medium and those in which the permeability is lower than in the surrounding medium. In a medium with the former type of fracture the fluid has a tendancy to flow into the fracture and then to flow along the fracture. In this case, one should not expect the Darcy velocity to be identical on the two sides of the fracture. Consequently, as the fracture is treated as an interface, the normal component of the velocity need not be continuous across this interface. An earlier model described by Alboin, Jaffré and Roberts in [1] and [2] was based on the assumption that the permeability in the fracture was large and a jump in the normal component of the velocity across the interface was permitted.

However, when the fracture has a lower permeability, the fluid naturally tends to avoid the fracture, which represents in fact a geological barrier. Two geological layers separated by such a barrier have little communication. Thus, it is understandable that the pressure need not be the same on the two sides of the fracture. In this case the pressure is not continuous across the fracture-interface.

We present in this paper a model that generalizes the earlier model (see [1], [2]) so that it can handle both large and small permeability fractures. Indeed, the earlier model assumed the continuity of the pressure across the interface. This is no longer the case with the model presented here.

The model is derived through a process of averaging across the fracture. This process is carried out for the flow equation written in mixed form. One thereby obtains a flow equation along the interface, that is coupled with flow equations in the neighboring subdomains. The main difference between this model and the previous one is that here nonstandard Robin type conditions are imposed at the interface. The Robin coefficient is proportional to the ratio of the permeability in the fracture to the fracture width. A parameter is introduced yielding a family of models. Existence and uniqueness of the solution of the mixed weak formulation of the problem is proved for certain values of the parameter. An error estimate is obtained for a particular choice of the parameter. Some numerical experiments show the quality of the results.

The simplest form of our model was presented in [11]. Others have also treated fractures as interfaces: in (see [5]), Helmig et. al. represented the fracture by lower dimensional finite elements. A model presented by Angot in [3] is based on Robin boundary conditions at the interface and assume the continuity of the flux across the fracture. A model similar to ours for a certain value of the parameter was studied by Flauraud et al. in [9].

\section{Description of the problem}

We suppose that $\Omega$ is a convex domain in $\mathbb{R}^{n}, n=2$ or 3 , and we denote by $\Gamma=\partial \Omega$ the boundary of $\Omega$. We suppose that the flow in $\Omega$ is governed by a conservation equation together with Darcy's law relating the gradient of the pressure $p$ to the Darcy velocity $\mathbf{u}$ :

$$
\begin{aligned}
\operatorname{div} \mathbf{u} & =q & & \text { in } \Omega \\
\mathbf{u} & =-\mathbf{K} \nabla p & & \text { in } \Omega \\
p & =\bar{p} & & \text { on } \Gamma,
\end{aligned}
$$

where $p$ is the pressure, $\mathbf{u}$ the Darcy velocity, $\mathbf{K}$ the hydraulic conductivity (or permeability) tensor, $q$ a source term and $\bar{p}$ the given pressure on the boundary $\Gamma$. We suppose that $\mathbf{K}$ is diagonal and that each diagonal entry $K_{j j}, j=1,2, \ldots n$ is positive and bounded above and away from 0 :

$$
0<K_{\min } \leq K_{j j} \leq K_{\max } \quad j=1,2, \ldots n \quad \text { a.e. in } \Omega .
$$


We suppose (see Figure 1) that the fracture, $\Omega_{f}$, is a sub-domain of $\Omega$, that there is a hyperplane $\gamma$ and a unit vector $\mathbf{n}=\mathbf{n}_{\mathbf{1}}=-\mathbf{n}_{\mathbf{2}}$ normal to $\gamma$ such that

$$
\begin{aligned}
\Omega_{f}= & \{x \in \Omega: x=s+r \mathbf{n} \text { for some } s \in \gamma \\
& \text { and some } \left.r \text { in the interval }\left(-\frac{d(s)}{2}, \frac{d(s)}{2}\right)\right\}
\end{aligned}
$$

where $d(s)$ denotes the thickness of the fracture at $h \in \gamma$.

We also assume that $\bar{\Omega}_{f}$ separates $\Omega$ into two connected subdomains

$$
\Omega \backslash \bar{\Omega}_{f}=\Omega_{1} \cup \Omega_{2}, \quad \Omega_{1} \cap \Omega_{2}=\emptyset .
$$

We denote by $\Gamma_{i}$ the part of the boundary of $\Omega_{i}$ in common with the boundary of $\Omega, i=1,2, f$

$$
\Gamma_{i}=\partial \Omega_{i} \cap \Gamma, \quad i=1,2, f,
$$

and we denote by $\gamma_{i}$ the part of the boundary of $\Omega_{i}$ in common with the boundary of the fracture $\Omega_{f}, i=1,2$

$$
\gamma_{i}=\partial \Omega_{i} \cap \partial \Omega_{f} \cap \Omega, \quad i=1,2 .
$$

Let $\eta$ be the outward unit normal vector field on $\Gamma$.
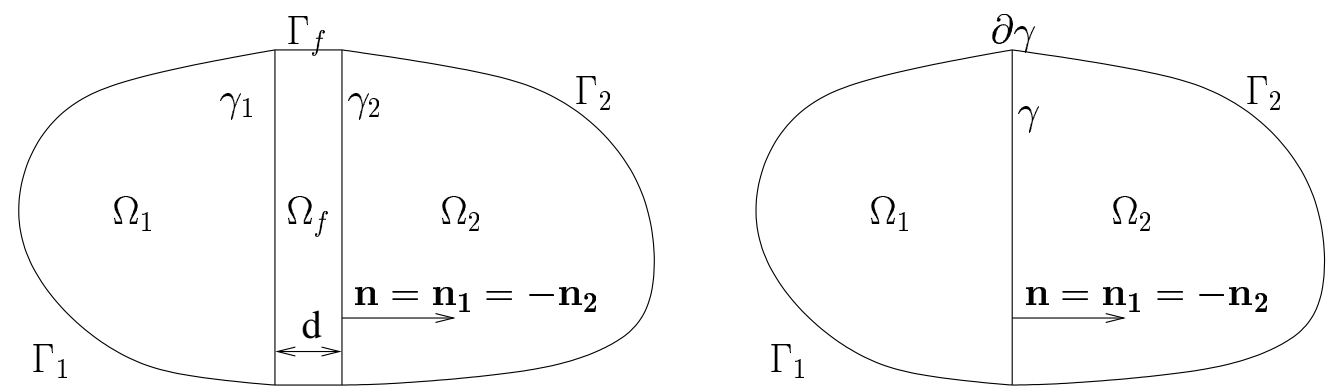

Figure 1: Left: the domain $\Omega$ with the fracture $\Omega_{f}$. Right: the subdomains $\Omega_{1}$ and $\Omega_{2}$ separated by the fracture considered as an interface $\gamma$.

If we denote by $p_{i}, \mathbf{u}_{i}, \mathbf{K}_{i}$, and $q_{i}$ the restrictions of $p, \mathbf{u}, \mathbf{K}$, and $q$ respectively to $\Omega_{i}, i=1,2, f$, and by $\bar{p}_{i}$ the restriction of $\bar{p}$ to $\Gamma_{i}, i=1,2, f$ we can rewrite the above problem (1) as a transmission problem:

$$
\begin{aligned}
\operatorname{div} \mathbf{u}_{i} & =q_{i} & & \text { in } \Omega_{i}, \quad i=1,2, f \\
\mathbf{u}_{i} & =-\mathbf{K}_{i} \boldsymbol{\nabla} p_{i} & & \text { in } \Omega_{i}, \quad i=1,2, f, \\
p_{i} & =\bar{p}_{i} & & \text { on } \Gamma_{i}, \quad i=1,2, f \\
p_{i} & =p_{f} & & \text { on } \gamma_{i}, \quad i=1,2, \\
\mathbf{u}_{i} \cdot \mathbf{n} & =\mathbf{u}_{f} \cdot \mathbf{n} & & \text { on } \gamma_{i}, \quad i=1,2 .
\end{aligned}
$$

\section{Derivation of the model}

In the model presented here the fracture is treated as an interface between the domains $\Omega_{1}$ and $\Omega_{2}$. The model is obtained by averaging along the line segments $[\mathbf{s}-d(\mathbf{s}) \mathbf{n}, \mathbf{s}+d(\mathbf{s}) \mathbf{n}], \mathbf{s} \in \gamma$, normal to $\gamma$. Treatment of the conservation equation is straightforward: there results a conservation equation on the surface $\gamma$ with a source term representing flow into the fracture from the subdomains. Darcy's law is a vector equation and averaging the components tangential to $\gamma$ yields a Darcy law in $\gamma$ relating the tangential component of the gradient of the averaged pressure to the tangential component of the 
averaged Darcy velocity. The system of these two equations in $\gamma$ is then of the same form as the system in each of the subdomains, only we have here a source term representing the exchange between $\gamma$ and the subdomains. The remaining equation involving the normal components of the vectors in Darcy's law, must be exploited to obtain boundary conditions along $\gamma$ for the systems in $\Omega_{1}$ and $\Omega_{2}$; however, averaging this equation yields only a formula for the difference of the trace along $\gamma$ of the pressure in $\Omega_{1}$ and that of the pressure in $\Omega_{2}$. Several possibilities exist for closing the system.

\subsection{Averaging across the fracture}

First decompose $\mathbf{u}_{\mathbf{f}}$ as $\mathbf{u}_{\mathbf{f}}=\mathbf{u}_{f, \mathbf{n}}+\mathbf{u}_{f, \tau}$ with $\mathbf{u}_{f, \mathbf{n}}=\mathbf{u}_{\mathbf{f}} \cdot \mathbf{n} \mathbf{n}$ (recall that that $\mathbf{n}=\mathbf{n}_{\mathbf{1}}=-\mathbf{n}_{\mathbf{2}}$ ). Let $\boldsymbol{\nabla}_{\tau}$ and $\operatorname{div}_{\tau}$ denote the tangential gradient and divergence operators and $\boldsymbol{\nabla}_{\mathbf{n}}$ and $\operatorname{div}_{\mathbf{n}}$ the normal gradient divergence operators.

\subsubsection{Averaging the conservation equation}

With the above notation, the first equation of (2) for $i=f$ may be rewritten as

$$
\operatorname{div}_{\mathbf{n}} \mathbf{u}_{f}+\operatorname{div}_{\tau} \mathbf{u}_{f}=q_{f} \quad \text { in } \Omega_{f}
$$

Integrating in the direction normal to the fracture one obtains

$$
\left.\mathbf{u}_{f} \cdot \mathbf{n}\right|_{\gamma_{2}}-\left.\mathbf{u}_{f} \cdot \mathbf{n}\right|_{\gamma_{1}}+\operatorname{div}_{\tau} \mathbf{U}_{f}=Q_{f} \quad \text { on } \gamma
$$

where $\mathbf{U}_{f}=\int_{-d / 2}^{d / 2} \mathbf{u}_{f, \tau} d \mathbf{n}$ and $Q_{f}=\int_{-d / 2}^{d / 2} q_{f} d \mathbf{n}$. Then using the continuity of the fluxes across $\gamma_{1}$ and $\gamma_{2}$, the last equation of (2) for $i=1$ and 2 , we may write

$$
\operatorname{div}_{\tau} \mathbf{U}_{f}=Q_{f}+\left(\left.\mathbf{u}_{1} \cdot \mathbf{n}_{1}\right|_{\gamma_{1}}+\left.\mathbf{u}_{2} \cdot \mathbf{n}_{2}\right|_{\gamma_{2}}\right) \quad \text { on } \gamma .
$$

This is the conservation equation on $\gamma$ with the additional source term $\left.\mathbf{u}_{1} \cdot \mathbf{n}_{1}\right|_{\gamma_{1}}+\left.\mathbf{u}_{2} \cdot \mathbf{n}_{2}\right|_{\gamma_{2}}$.

\subsubsection{Averaging Darcy's law}

The second equation of (2) for $i=f$ may be written

$$
\begin{aligned}
& \mathbf{u}_{f, \tau}=-\mathbf{K}_{f, \tau} \boldsymbol{\nabla}_{\tau} p_{f} \quad \text { in } \Omega_{f} \\
& \mathbf{u}_{f, \mathbf{n}}=-\mathbf{K}_{f, \mathbf{n}} \boldsymbol{\nabla}_{\mathbf{n}} p_{f} \quad \text { in } \Omega_{f} .
\end{aligned}
$$

Again, integrating in the direction normal to the fracture, one obtains from the first equation of (6)

$$
\mathbf{U}_{f}=-\mathbf{K}_{f, \tau} d \boldsymbol{\nabla}_{\tau} P_{f} \quad \text { on } \gamma
$$

where $P_{f}=\frac{1}{d} \int_{-d / 2}^{d / 2} p_{f} d \mathbf{n}$ and where we have assumed that $\mathbf{K}_{f, \tau}$ is constant along the segments $[\mathbf{s}-d(\mathbf{s}) \mathbf{n}, \mathbf{s}+d(\mathbf{s}) \mathbf{n}]$. This is Darcy's law in the $(n-1)$-dimensional domain $\gamma$. Together (5) and (7) give a flow equation in $\gamma$ with a source term representing the flow from the subdomains $\Omega_{1}$ and $\Omega_{2}$ into the fracture. The remaining equation, the second equation of (6) must now be used to give boundary conditions along $\gamma$ for the systems in $\Omega_{1}$ and $\Omega_{2}$ which allow for a pressure difference from one side of $\gamma$ to the other.

Integrating the remaining equation, the second equation (6), in the direction normal to the fracture one obtains

$$
\int_{-d / 2}^{d / 2} \mathbf{u}_{f, \mathbf{n}} \cdot \mathbf{n} d \mathbf{n}=-\mathbf{K}_{f, \mathbf{n}}\left(p_{f \mid \gamma_{2}}-p_{f \mid \gamma_{1}}\right) .
$$


The integral $\int_{-d / 2}^{d / 2} \mathbf{u}_{f, \mathbf{n}} \cdot \mathbf{n} d \mathbf{n}$ has not been computed but can be approximated using the trapezoidal rule:

$$
\int_{-d / 2}^{d / 2} \mathbf{u}_{f, \mathbf{n}} \cdot \mathbf{n} d \mathbf{n} \approx \frac{d}{2}\left(\left.\mathbf{u}_{f} \cdot \mathbf{n}\right|_{\gamma_{2}}+\left.\mathbf{u}_{f} \cdot \mathbf{n}\right|_{\gamma_{1}}\right)=\frac{d}{2}\left(\left.\mathbf{u}_{2} \cdot \mathbf{n}\right|_{\gamma_{2}}+\left.\mathbf{u}_{1} \cdot \mathbf{n}\right|_{\gamma_{1}}\right),
$$

where we have used the continuity along $\gamma_{1}$ and $\gamma_{2}$ of the fluxes, the fifth equation of (2). Now using the continuity along $\gamma_{1}$ and $\gamma_{2}$ of the pressures, the fourth equation of (2), the second equation of (6) is approximated by

$$
\frac{1}{2}\left(\left.\mathbf{u}_{2} \cdot \mathbf{n}\right|_{\gamma_{2}}+\left.\mathbf{u}_{1} \cdot \mathbf{n}\right|_{\gamma_{1}}\right)=-\mathbf{K}_{f, \mathbf{n}} \frac{p_{2} \mid \gamma_{2}-p_{1 \mid \gamma_{1}}}{d}
$$

or using the notation

$$
\begin{gathered}
\alpha_{f}=\frac{2 \mathbf{K}_{f, \mathbf{n}}}{d} \\
-\left.\mathbf{u}_{1} \cdot \mathbf{n}_{1}\right|_{\gamma_{1}}+\alpha_{f} p_{1 \mid \gamma_{1}}=-\left.\mathbf{u}_{2} \cdot \mathbf{n}_{2}\right|_{\gamma_{2}}+\alpha_{f} p_{2 \mid \gamma_{2}} .
\end{gathered}
$$

This gives an equation for the pressure difference across the fracture.

\section{Boundary conditions along $\gamma, \mathbf{I}$}

To close the system and obtain boundary conditions on $\gamma$ for the problems in $\Omega_{1}$ and $\Omega_{2}$ one last equation is necessary. As (9) gives the difference between the pressures across $\gamma$, a natural choice is to obtain an equation for the sum of the pressures on $\gamma$ by supposing that the average pressure across the fracture $P_{f}$ is also the average of the pressures on the boundaries $\gamma_{1}$ and $\gamma_{2}$. Thus to calculate $p_{2} \mid \gamma_{2}$ and $p_{1 \mid \gamma_{1}}$, we use the two equations

$$
\begin{aligned}
& p_{2 \mid \gamma_{2}}-p_{1 \mid \gamma_{1}}=\frac{d}{2 K_{f}}\left(\left.\mathbf{u}_{2} \cdot \mathbf{n}_{2}\right|_{\gamma_{2}}-\left.\mathbf{u}_{1} \cdot \mathbf{n}_{1}\right|_{\gamma_{1}}\right) \\
& p_{2 \mid \gamma_{2}}+p_{1 \mid \gamma_{1}}=2 P_{f} .
\end{aligned}
$$

Summing and subtracting the two equations in (11), one gets

$$
\begin{aligned}
& -1 /\left.2 \mathbf{u}_{1} \cdot \mathbf{n}_{1}\right|_{\gamma_{1}}+\left.\alpha_{f} p_{1}\right|_{\gamma_{1}}=-1 /\left.2 \mathbf{u}_{2} \cdot \mathbf{n}_{2}\right|_{\gamma_{2}}+\alpha_{f} P_{f} \\
& -1 /\left.2 \mathbf{u}_{2} \cdot \mathbf{n}_{2}\right|_{\gamma_{2}}+\left.\alpha_{f} p_{2}\right|_{\gamma_{2}}=-1 /\left.2 \mathbf{u}_{1} \cdot \mathbf{n}_{1}\right|_{\gamma_{1}}+\alpha_{f} P_{f} .
\end{aligned}
$$

\section{Boundary conditions along $\gamma$, II}

If we approximate the value of $p_{f}$ at the center of the fracture by $P_{f}$, and the value of $\left(\mathbf{u}_{f} \cdot \mathbf{n}\right)$ at the center of the fracture by the averaged flux, $\frac{\mathbf{u}_{f} \cdot \mathbf{n}_{f}\left|\gamma_{1}+\mathbf{u}_{f} \cdot \mathbf{n}_{f}\right| \gamma_{2}}{2}$, and then average across each half of the fracture in the same way that we averaged across the entire width of the fracture above we obtain the following 2 equations:

or

$$
\begin{aligned}
& \frac{1}{4}\left(\left.3 \mathbf{u}_{2} \cdot \mathbf{n}\right|_{\gamma_{2}}+\left.\mathbf{u}_{1} \cdot \mathbf{n}\right|_{\gamma_{1}}\right)=-\mathbf{K}_{f, \mathbf{n}} \frac{p_{f \mid \gamma_{2}}-P_{f}}{d / 2} \\
& \frac{1}{4}\left(\left.\mathbf{u}_{2} \cdot \mathbf{n}\right|_{\gamma_{2}}+\left.3 \mathbf{u}_{1} \cdot \mathbf{n}\right|_{\gamma_{1}}\right)=-\mathbf{K}_{f, \mathbf{n}} \frac{P_{f}-p_{f \mid \gamma_{1}}}{d / 2}
\end{aligned}
$$

$$
\begin{aligned}
& -3 /\left.4 \mathbf{u}_{1} \cdot \mathbf{n}_{1}\right|_{\gamma_{1}}+\left.\alpha_{f} p_{1}\right|_{\gamma_{1}}=-1 /\left.4 \mathbf{u}_{2} \cdot \mathbf{n}_{2}\right|_{\gamma_{2}}+\alpha_{f} P_{f} \\
& -3 /\left.4 \mathbf{u}_{2} \cdot \mathbf{n}_{2}\right|_{\gamma_{2}}+\left.\alpha_{f} p_{2}\right|_{\gamma_{2}}=-1 /\left.4 \mathbf{u}_{1} \cdot \mathbf{n}_{1}\right|_{\gamma_{1}}+\alpha_{f} P_{f} .
\end{aligned}
$$

These equations can be subtracted to recover (9), or the first equation of (11), but addition yields not the second equation of (11) but (after dividing by $d / 2$ )

$$
-K_{f} \frac{p_{2 \mid \gamma_{2}}+p_{1 \mid \gamma_{1}}-2 P_{f}}{d^{2} / 4}=\frac{\left.\mathbf{u}_{2} \cdot \mathbf{n}\right|_{\gamma_{2}}-\left.\mathbf{u}_{1} \cdot \mathbf{n}\right|_{\gamma_{1}}}{d} .
$$


The two equations of (14) can also be combined to obtain

$$
\begin{aligned}
& \left.\mathbf{u}_{1} \cdot \mathbf{n}_{1}\right|_{\gamma_{1}}=-\frac{K_{f}}{d}\left(4 P_{f}-\left.3 p_{1}\right|_{\gamma_{1}}-\left.p_{2}\right|_{\gamma_{2}}\right) \\
& \left.\mathbf{u}_{2} \cdot \mathbf{n}_{2}\right|_{\gamma_{2}}=-\frac{K_{f}}{d}\left(4 P_{f}-\left.p_{1}\right|_{\gamma_{1}}-\left.3 p_{2}\right|_{\gamma_{2}}\right) .
\end{aligned}
$$

\section{Boundary conditions along $\gamma$, III}

The equations (16) suggest another possibility:

$$
\begin{aligned}
& \left.\mathbf{u}_{1} \cdot \mathbf{n}_{1}\right|_{\gamma_{1}}=-\mathbf{K}_{f} \frac{\left.p_{f}\right|_{\gamma_{1}}-P_{f}}{d / 2} \\
& \left.\mathbf{u}_{2} \cdot \mathbf{n}_{2}\right|_{\gamma_{2}}=-\mathbf{K}_{f} \frac{\left.p_{f}\right|_{\gamma_{2}}-P_{f}}{d / 2} .
\end{aligned}
$$

or, using the coefficient $\alpha_{f}$ defined in (10),

$$
\begin{aligned}
& -\left.\mathbf{u}_{1} \cdot \mathbf{n}_{1}\right|_{\gamma_{1}}+\left.\alpha_{f} p_{1}\right|_{\gamma_{1}}=-\alpha_{f} P_{f} \\
& -\left.\mathbf{u}_{2} \cdot \mathbf{n}_{2}\right|_{\gamma_{2}}+\left.\alpha_{f} p_{2}\right|_{\gamma_{2}}=-\alpha_{f} P_{f}
\end{aligned}
$$

From these two equations we can again recover (9) or the first equation of (11) but the second equation of (11) is replaced by

$$
\begin{aligned}
& -K_{f} \frac{p_{2 \mid \gamma_{2}}+p_{1 \mid \gamma_{1}}-2 P_{f}}{d^{2} / 2}=\frac{\left.\mathbf{u}_{2} \cdot \mathbf{n}\right|_{\gamma_{2}}-\left.\mathbf{u}_{1} \cdot \mathbf{n}\right|_{\gamma_{1}}}{d} \\
& \text { or } \\
& p_{2 \mid \gamma_{2}}+p_{1 \mid \gamma_{1}}=2 P_{f}-\frac{d}{2 K_{f}}\left(\left.\mathbf{u}_{2} \cdot \mathbf{n}\right|_{\gamma_{2}}-\left.\mathbf{u}_{1} \cdot \mathbf{n}\right|_{\gamma_{1}}\right),
\end{aligned}
$$

\section{Boundary conditions along $\gamma$, the general case}

The three sets of equation (12), (14), and (18) can be written in the form

$$
\begin{aligned}
-\left.\xi \mathbf{u}_{1} \cdot \mathbf{n}_{1}\right|_{\gamma_{1}}+\left.\alpha_{f} p_{1}\right|_{\gamma_{1}} & =-\left.(1-\xi) \mathbf{u}_{2} \cdot \mathbf{n}_{2}\right|_{\gamma_{2}}+\alpha_{f} P_{f} \\
-\left.\xi \mathbf{u}_{2} \cdot \mathbf{n}_{2}\right|_{\gamma_{2}}+\left.\alpha_{f} p_{2}\right|_{\gamma_{2}} & =-\left.(1-\xi) \mathbf{u}_{1} \cdot \mathbf{n}_{1}\right|_{\gamma_{1}}+\alpha_{f} P_{f}
\end{aligned}
$$

for the values $\xi=1 / 2, \xi=3 / 4$ and $\xi=1$ respectively.

\section{Model problem for the fracture}

We study the following model problem which is an extension of the previous work (see [2], [1]).

\subsection{Strong formulation of the model problem}

The parameter $\xi$ is a positive real number that will be determined later on. For common applications, this parameter should be such that: $\xi \in] 1 / 2,1]$. We use the notation $\nabla_{\tau}$ (and respectively $\operatorname{div}_{\tau}$ ) for the tangential gradient (and respectively tangential divergence) operators along the fracture $\gamma$. We assume that the index $i$ of the subdomains varies in $Z / 2 Z$ (so that $2+1=1$ ). Posing $\alpha_{f}=\frac{2 \mathbf{K}_{f, \mathbf{n}}}{d}$, the problem can be written

$$
\begin{aligned}
\mathbf{u}_{i} & =-\mathbf{K}_{i} \boldsymbol{\nabla} p_{i} & & \text { in } \Omega_{i}, \quad i=1,2 \\
\operatorname{div} \mathbf{u}_{i} & =q_{i} & & \text { in } \Omega_{i}, \quad i=1,2 \\
\mathbf{u}_{f}^{\tau} & =-\mathbf{K}_{f, \tau} d \boldsymbol{\nabla}_{\tau} p_{f} & & \text { in } \gamma \\
\operatorname{div}_{\tau} \mathbf{u}_{f}^{\tau} & =q_{f}+\left(\left.\mathbf{u}_{\mathbf{1}} \cdot \mathbf{n}_{\mathbf{1}}\right|_{\gamma}+\left.\mathbf{u}_{\mathbf{2}} \cdot \mathbf{n}_{\mathbf{2}}\right|_{\gamma}\right) & & \text { in } \gamma \\
-\xi \mathbf{u}_{\mathbf{i}} \cdot \mathbf{n}_{\mathbf{i}}+\alpha_{f} p_{i} & =\alpha_{f} p_{f}-(1-\xi) \mathbf{u}_{\mathbf{i}+\mathbf{1}} \cdot \mathbf{n}_{\mathbf{i}+\mathbf{1}} & & \text { in } \gamma, \quad i=1,2 \\
p_{i} & =\bar{p}_{i} & & \text { on } \Gamma_{i}, \quad i=1,2 . \\
p_{f} & =\bar{p}_{f} & & \text { on } \partial \gamma .
\end{aligned}
$$


This system can be seen as a domain decomposition problem, with nonstandard and nonlocal boundary conditions between the subdomains. The third equation in (22) represents Darcy's law in the direction tangential to the fracture. The fourth equation in (22) models mass conservation inside the fracture. A source term $\left(\left.\mathbf{u}_{\mathbf{1}} \cdot \mathbf{n}_{\mathbf{1}}\right|_{\gamma_{1}}+\left.\mathbf{u}_{\mathbf{2}} \cdot \mathbf{n}_{\mathbf{2}}\right|_{\gamma_{2}}\right)$ is introduced to take into account the contribution of the subdomain flows to the fracture flow. The fifth equation in (22) is a Robin boundary condition for the subdomain $\Omega_{i}$ with a dependence with the pressure in the fracture $p_{f}$ and also with neighboring subdomain $\Omega_{i+1}$ 's fluxes. This last dependence only disappears when $\xi$ is equal to 1 , and this case will be seen to be important for the below domain decomposition formulation (see Section 7).

This fifth equation in (22) is equivalent to

$$
\left.p_{i}\right|_{\gamma}=p_{f}+\frac{\xi}{\alpha_{f}} \mathbf{u}_{\mathbf{i}} \cdot \mathbf{n}_{\mathbf{i}}-\frac{1-\xi}{\alpha_{f}} \mathbf{u}_{\mathbf{i}+\mathbf{1}} \cdot \mathbf{n}_{\mathbf{i}+\mathbf{1}} \quad \text { on } \gamma, \quad i=1,2 .
$$

It is in this form that Robin boundary conditions are expressed in mixed formulation. If $\xi$ is greater than $1 / 2$, we can also write

$$
\begin{aligned}
& \left.\mathbf{u}_{2} \cdot \mathbf{n}_{2}\right|_{\gamma_{2}}+\left.\mathbf{u}_{1} \cdot \mathbf{n}_{1}\right|_{\gamma_{1}}=\frac{\alpha_{f}}{2 \xi-1}\left(\left.p_{2}\right|_{\gamma_{2}}+\left.p_{1}\right|_{\gamma_{1}}-2 p_{f}\right), \\
& \left.\mathbf{u}_{2} \cdot \mathbf{n}_{2}\right|_{\gamma_{2}}-\left.\mathbf{u}_{1} \cdot \mathbf{n}_{1}\right|_{\gamma_{1}}=\alpha_{f}\left(\left.p_{2}\right|_{\gamma_{2}}-\left.p_{1}\right|_{\gamma_{1}}\right) .
\end{aligned}
$$

Remarks: The difference between this model and the model with pressure continuous across the $(n-1)$ dimensional fracture (see [1], [2]) is that in the earlier model equations (23) are replaced by $p_{2 \mid \gamma_{2}}=$ $p_{1 \mid \gamma_{1}}=p_{f}$. This corresponds to the assumption $\frac{1}{\alpha_{f}}=\frac{d}{2 K_{f}} \approx 0$ that was made previously (large permeability and small width in the fracture).

Remark: The model depends actually on two physical, fracture dependent coefficients: the product $\mathbf{K}_{f, \tau} d$ and the ratio $\mathbf{K}_{f, \mathbf{n}} / d$. The first coefficient is related to the jump in the normal component of the velocity (non continuity of the Darcy velocity across the fracture). The second one is related to the pressure jump (non continuity of pressure across the fracture).

The coefficient $\mathbf{K}_{f, \tau} d$ represents the equivalent permeability for the flow along the fracture. When this coefficient is of the same order as the permeability in the other subdomains $\left(\mathbf{K}_{f, \tau} d \approx K_{i}, i=1,2\right)$, i.e. when the fracture permeability is sufficiently large, the flow along the fracture interacts with the flow in the rocks. In this case, the jump of the Darcy velocity across the fracture $\left(\left.\mathbf{u}_{\mathbf{1}} \cdot \mathbf{n}_{\mathbf{1}}\right|_{\gamma_{1}}+\left.\mathbf{u}_{\mathbf{2}} \cdot \mathbf{n}_{\mathbf{2}}\right|_{\gamma_{2}}\right)$ is generally not zero, because it is the contribution of the rock flow to the fracture flow. This result is in agreement with the asymptotic analysis study by Sanchez-Palencia and al. [10], where the fracture permeability was large. One example of this type of behaviour is shown in Section 6.1.

The coefficient $\mathbf{K}_{f, \mathbf{n}} / d\left(=\alpha_{f} / 2\right)$ represents an equivalent "resistivity" across the fracture. Let us assume that the fracture permeability is small and that the coefficient is of the same order as the permeability in the subdomains $\left(\mathbf{K}_{f, \mathbf{n}} / d \approx K_{i}, i=1,2\right)$. In this case, the fluid barely flows along the fracture and the normal velocity jump is almost zero. Equations $(24,25)$ become, after dividing the second equation by 2 , equations $(26,27)$, which are similar to the transmission equations of [3]. This result is also in agreement with the asymptotic analysis study by Sanchez-Palencia [16], where the fracture permeability was small. One example of this type of behaviour is shown in Section 6.2.

$$
\begin{aligned}
\left.\mathbf{u}_{2} \cdot \mathbf{n}_{2}\right|_{\gamma_{2}}+\left.\mathbf{u}_{1} \cdot \mathbf{n}_{1}\right|_{\gamma_{1}} & =0 \\
\left.\mathbf{u}_{2} \cdot \mathbf{n}_{2}\right|_{\gamma_{2}} & =\frac{\mathbf{K}_{f, \mathbf{n}}}{d}\left(\left.p_{2}\right|_{\gamma_{2}}-\left.p_{1}\right|_{\gamma_{1}}\right) .
\end{aligned}
$$

\subsection{Weak formulation of the model problem}

We will need the following Hilbert spaces $M$ and $\mathbf{W}$. It is necessary to assume more regularity than the $\mathcal{H}(\operatorname{div}, *)$ regularity (used commonly for the Mixed Finite element methods), in order to take into 
account properly the Robin boundary conditions (see [15] pp. 589-590 for instance).

$$
\begin{aligned}
M= & \left\{r=\left(r_{1}, r_{2}, r_{\gamma}\right) \in L^{2}\left(\Omega_{1}\right) \times L^{2}\left(\Omega_{2}\right) \times L^{2}(\gamma)\right\} \\
\mathbf{W}= & \left\{\mathbf{v}=\left(\mathbf{v}_{\mathbf{1}}, \mathbf{v}_{\mathbf{2}}, \mathbf{v}_{\mathbf{f}}\right) \in \mathcal{H}\left(\operatorname{div}, \Omega_{1}\right) \times \mathcal{H}\left(\operatorname{div}, \Omega_{2}\right) \times \mathcal{H}\left(\operatorname{div}_{\tau}, \gamma\right):\right. \\
& \left.\mathbf{v}_{\mathbf{i}} \cdot \mathbf{n}_{\mathbf{i}} \in L^{2}(\gamma), \quad i=1,2\right\}
\end{aligned}
$$

equipped with the norms

$$
\begin{aligned}
\|r\|_{M}^{2} & =\sum_{i=1}^{2}\left\|r_{i}\right\|_{0, \Omega_{i}}^{2}+\left\|r_{f}\right\|_{0, \gamma}^{2} \\
\|\mathbf{v}\|_{W}^{2} & =\sum_{i=1}^{2}\left(\left\|\mathbf{v}_{\mathbf{i}}\right\|_{0, \Omega_{i}}^{2}+\left\|\operatorname{div} \mathbf{v}_{\mathbf{i}}\right\|_{0, \Omega_{i}}^{2}\right)+\left\|\mathbf{v}_{\mathbf{f}}\right\|_{0, \gamma}^{2}+\left\|\operatorname{div}_{\tau} \mathbf{v}_{\mathbf{f}}\right\|_{0, \gamma}^{2}+\sum_{i=1}^{2}\left\|\mathbf{v}_{\mathbf{i}} \cdot \mathbf{n}_{\mathbf{i}}\right\|_{0, \gamma}^{2} .
\end{aligned}
$$

Let the bilinear forms $\alpha_{\xi}: \mathbf{W} \times \mathbf{W} \rightarrow \mathbb{R}$ and $\beta: \mathbf{W} \times M \rightarrow \mathbb{R}$ be defined by

$$
\begin{aligned}
\alpha_{\xi}(\mathbf{u}, \mathbf{v}) & =\sum_{i=1}^{2}\left(K_{i}^{-1} \mathbf{u}_{\mathbf{i}}, \mathbf{v}_{\mathbf{i}}\right)_{\Omega_{i}}+\left(\left(K_{f, \tau} d\right)^{-1} \mathbf{u}_{\mathbf{f}}, \mathbf{v}_{\mathbf{f}}\right)_{\gamma} \\
& +\sum_{i=1}^{2}\left(\frac{1}{\alpha_{f}}\left[\xi \mathbf{u}_{\mathbf{i}} \cdot \mathbf{n}_{\mathbf{i}}-(1-\xi) \mathbf{u}_{\mathbf{i}+\mathbf{1}} \cdot \mathbf{n}_{\mathbf{i}+\mathbf{1}}\right], \mathbf{v}_{\mathbf{i}} \cdot \mathbf{n}_{\mathbf{i}}\right)_{\gamma}, \\
\beta(\mathbf{u}, r) & =\sum_{i=1}^{2}\left(\operatorname{div} \mathbf{u}_{\mathbf{i}}, r_{i}\right)_{\Omega_{i}}+\left(\operatorname{div}_{\tau} \mathbf{u}_{\mathbf{f}}, r_{f}\right)_{\gamma}-\left(\sum_{i=1}^{2} \mathbf{u}_{\mathbf{i}} \cdot \mathbf{n}_{\mathbf{i}}, r_{f}\right)_{\gamma}
\end{aligned}
$$

Let the linear forms $L_{q}: M \rightarrow \mathbb{R}$ and $L_{d}: \mathbf{W} \rightarrow \mathbb{R}$ be defined by

$$
\begin{aligned}
L_{q}(r) & =\sum_{i=1}^{2}\left(q_{i}, r_{i}\right)_{\Omega_{i}}+\left(q_{f}, r_{f}\right)_{\gamma}, \\
L_{d}(\mathbf{v}) & =\sum_{j=1}^{2}\left(\mathbf{v}_{\mathbf{j}} \cdot \mathbf{n}_{\mathbf{j}}, \bar{p}_{j}\right)_{\Gamma_{j}}+\left(\mathbf{v}_{\mathbf{f}} \cdot \mathbf{n}_{\mathbf{f}}, \bar{p}_{f}\right)_{\partial \gamma} .
\end{aligned}
$$

With these spaces and forms, one can easily see that the weak form of (22) may be written as follows:

$$
\begin{aligned}
& \mathbf{u} \in \mathbf{W}, p \in M \\
& \alpha_{\xi}(\mathbf{u}, \mathbf{v})-\beta(\mathbf{v}, p)=-L_{d}(\mathbf{v}) \quad \forall \mathbf{v} \in \mathbf{W} \\
& \beta(\mathbf{u}, r) \quad=L_{q}(r) \quad \forall r \in M \text {. }
\end{aligned}
$$

\subsection{Existence and uniqueness of the solution}

We assume that the right-hand side $q$ is sufficiently regular (in $M$ for instance) and for simplicity that there are homogeneous Dirichlet boundary conditions imposed $\left(L_{d}=0\right)$. The domain and the fracture are also assumed to be smooth enough. Then we can state the following existence theorem:

Theorem 1 Assume that there exists two positive constants $0<K_{\min }<K_{\max }$, such that the permeabilities in the two subdomains and the coefficients $\left(\mathbf{K}_{f, \tau} d\right)$ and $\left(\mathbf{K}_{f, \mathbf{n}} / d\right)=\alpha_{f} / 2$ are bounded by these constants: $0<K_{\min } \leq K_{i} \leq K_{\max }, i=1,2$, and $0<K_{\min } \leq\left(\mathbf{K}_{f, \tau} d\right) \leq K_{\max }$, and

$0<K_{\min } \leq\left(\mathbf{K}_{f, \mathbf{n}} / d\right) \leq K_{\max }$. Assume also that the parameter $\xi>1 / 2$. Then the model problem (28) has a unique solution.

Proof: It is easy to check that $\mathbf{W}$ and $M$ are Hilbert spaces (see [15] pp. 530). The continuity of $\alpha_{\xi}$ over $\mathbf{W}^{2}$ and of $\beta$ over $\mathbf{W} \times M$ is straighforward. 
We introduce the subspace $\tilde{\mathbf{W}}=\{\mathbf{v} \in \mathbf{W}: \beta(\mathbf{v}, r)=0 \quad \forall r \in M\}$. To show existence and uniqueness of the solution of (28), it is sufficient to show that $\alpha_{\xi}$ is $\tilde{\mathbf{W}}$-elliptic and that $\beta$ satisfies the inf-sup condition (see $[15,6]$ ), that is there exist constants $C_{\alpha}$ and $C_{\beta}$ such that

$$
\inf _{\mathbf{v} \in \tilde{\mathbf{W}}} \frac{\alpha_{\xi}(\mathbf{v}, \mathbf{v})}{\|\mathbf{v}\|_{\mathbf{W}}^{2}} \geq C_{\alpha}, \quad \inf _{r \in M} \sup _{\mathbf{v} \in \mathbf{W}} \frac{\beta(\mathbf{v}, r)}{\|r\|_{M}\|\mathbf{v}\|_{\mathbf{W}}} \geq C_{\beta}
$$

To check that $\alpha_{\xi}$ is $\tilde{\mathbf{W}}$-elliptic, we note that for $\mathbf{u} \in \tilde{\mathbf{W}},\left\|\operatorname{div} \mathbf{u}_{\mathbf{i}}\right\|_{0, \Omega_{i}}=0$ and $\operatorname{div}_{\tau} \mathbf{u}_{\mathbf{f}}=\mathbf{u}_{\mathbf{1}} \cdot \mathbf{n}_{\mathbf{1}}+$ $\mathbf{u}_{2} \cdot \mathbf{n}_{2}$. Thus

$$
\|\mathbf{u}\|_{\mathbf{W}}^{2}=\sum_{i=1}^{2}\left\|\mathbf{u}_{i}\right\|_{0, \Omega_{i}}^{2}+\left\|\mathbf{u}_{\mathbf{f}}\right\|_{0, \gamma}^{2}+\left\|\sum_{i=1}^{2} \mathbf{u}_{\mathbf{i}} \cdot \mathbf{n}_{\mathbf{i}}\right\|_{0, \gamma}^{2}+\sum_{i=1}^{2}\left\|\mathbf{u}_{\mathbf{i}} \cdot \mathbf{n}_{\mathbf{i}}\right\|_{0, \gamma}^{2} .
$$

For this $\mathbf{u} \in \tilde{\mathbf{W}}, \alpha_{\xi}$ writes

$$
\begin{aligned}
\alpha_{\xi}(\mathbf{u}, \mathbf{u})= & \sum_{i=1}^{2}\left(K_{i}^{-1} \mathbf{u}_{\mathbf{i}}, \mathbf{u}_{\mathbf{i}}\right)_{\Omega_{i}}+\left(\left(K_{f, \tau} d\right)^{-1} \mathbf{u}_{\mathbf{f}}, \mathbf{u}_{\mathbf{f}}\right)_{\gamma} \\
& +\xi \sum_{i=1}^{2}\left(\frac{\mathbf{u}_{\mathbf{i}} \cdot \mathbf{n}_{\mathbf{i}}}{\alpha_{f}^{1 / 2}}, \frac{\mathbf{u}_{\mathbf{i}} \cdot \mathbf{n}_{\mathbf{i}}}{\alpha_{f}^{1 / 2}}\right)_{\gamma}-2(1-\xi)\left(\frac{\mathbf{u}_{\mathbf{1}} \cdot \mathbf{n}_{\mathbf{1}}}{\alpha_{f}^{1 / 2}}, \frac{\mathbf{u}_{\mathbf{2}} \cdot \mathbf{n}_{\mathbf{2}}}{\alpha_{f}^{1 / 2}}\right)_{\gamma} .
\end{aligned}
$$

The two first terms in equation (29) are easily estimated because the product $\left(\mathbf{K}_{f, \tau} d\right)$ and the permeabilities are bounded. Introducing $\phi_{i}=\alpha_{f}^{-1 / 2} \mathbf{u}_{\mathbf{i}} \cdot \mathbf{n}_{\mathbf{i}}, i=1,2$, the two other terms are equal to the quadratic form $B\left(\phi_{1}, \phi_{2}\right)=\xi\left\|\phi_{1}\right\|_{0, \gamma}^{2}-2(1-\xi)\left(\phi_{1}, \phi_{2}\right)_{\gamma}+\xi\left\|\phi_{2}\right\|_{0, \gamma}^{2}$. The eigenvalues of $B$ are 1 and $2 \xi-1$. Therefore $B$ is strictly elliptic if and only if $\xi>1 / 2$. In this case, we obtain the inequality

$$
\alpha_{\xi}(\mathbf{u}, \mathbf{u}) \geq K_{\max }^{-1}\left(\sum_{i=1}^{2}\left\|\mathbf{u}_{\mathbf{i}}\right\|_{0, \Omega_{i}}^{2}+\left\|\mathbf{u}_{\mathbf{f}}\right\|_{0, \gamma}^{2}\right)+\min \{1,2 \xi-1\} \sum_{i=1}^{2}\left\|\frac{\mathbf{u}_{\mathbf{i}} \cdot \mathbf{n}_{\mathbf{i}}}{\alpha_{f}^{1 / 2}}\right\|_{0, \gamma}^{2}
$$

As the ratio $\left(\mathbf{K}_{f, \mathbf{n}} / d\right)=\left(\alpha_{f} / 2\right)$ is bounded, and because $\left\|\sum_{i=1}^{2} \mathbf{u}_{\mathbf{i}} \cdot \mathbf{n}_{\mathbf{i}}\right\|_{0, \gamma}^{2} \leq 2 \sum_{i=1}^{2}\left\|\mathbf{u}_{\mathbf{i}} \cdot \mathbf{n}_{\mathbf{i}}\right\|_{0, \gamma}^{2}$, for $\xi>1 / 2$, we have

$$
\begin{aligned}
\alpha_{\xi}(\mathbf{u}, \mathbf{u}) & \geq K_{\max }^{-1}\left(\sum_{i=1}^{2}\left\|\mathbf{u}_{\mathbf{i}}\right\|_{0, \Omega_{i}}^{2}+\left\|\mathbf{u}_{\mathbf{f}}\right\|_{0, \gamma}^{2}+\min \{1,2 \xi-1\} \sum_{i=1}^{2}\left\|\mathbf{u}_{\mathbf{i}} \cdot \mathbf{n}_{\mathbf{i}}\right\|_{0, \gamma}^{2}\right) \\
& \geq 1 / 3 K_{\max }^{-1} \min \{1,2 \xi-1\}\|u\|_{\mathbf{W}}^{2} .
\end{aligned}
$$

To see that $\beta$ satisfies the inf-sup condition, given $r \in M$, we construct using the adjoint equation a $\mathbf{v} \in \mathbf{W}$ such that $\beta(\mathbf{v}, r)=\|r\|_{M}^{2}$ and $\|\mathbf{v}\|_{\mathbf{W}} \leq C\|r\|_{M}$, where $C$ is the constant of elliptic regularity for the adjoint problem.

For $r=\left(r_{1}, r_{2}, r_{\tau}\right) \in M$, let $\left(\varphi_{1}, \varphi_{2}, \varphi_{\gamma}\right) \in H^{2}\left(\Omega_{1}\right) \times H^{2}\left(\Omega_{2}\right) \times H^{2}(\gamma)$ be the solution of

$$
\begin{aligned}
-\Delta \varphi & =\tilde{r} \text { on } \Omega \\
\varphi & =0 \text { on } \Gamma,
\end{aligned}
$$

where $\tilde{r} \in L^{2}(\Omega)$ is given by $\tilde{r}_{\mid \Omega_{i}}=r_{i}, i=1,2$, and

$$
\begin{array}{rlll}
-\Delta_{\tau} \varphi_{\gamma} & =r_{\tau} & \text { on } \gamma \\
\varphi_{\gamma} & =0 & \text { on } \partial \gamma
\end{array}
$$

$\mathrm{RR} \mathrm{n}^{\circ} 4848$ 
Pose $\mathbf{v}_{\mathbf{i}}=-\boldsymbol{\nabla} \varphi_{\mid \Omega_{i}}, i=1,2$, and $\mathbf{v}_{\gamma}=-\boldsymbol{\nabla}_{\gamma} \varphi_{\gamma}$ and note that $\operatorname{div} \mathbf{v}_{i}=r_{i} \in L^{2}\left(\Omega_{i}\right), i=$ $1,2, \operatorname{div}_{\tau} \mathbf{v}_{\gamma}=r_{\tau} \in L^{2}(\gamma)$ and $\mathbf{v}_{\mathbf{1}} \cdot \mathbf{n}_{\mathbf{1}}=-\mathbf{v}_{\mathbf{2}} \cdot \mathbf{n}_{\mathbf{2}} \in L^{2}(\gamma)$, because $\mathbf{v}_{\mathbf{i}} \in\left(\mathcal{H}^{1}(\Omega)\right)^{d}$. Thus $\mathbf{v}=\left(\mathbf{v}_{1}, \mathbf{v}_{2}, \mathbf{v}_{\gamma}\right) \in \mathbf{W}$ and $\beta(\mathbf{v}, r)=\|\tilde{r}\|_{0, \Omega}^{2}+\left\|r_{f}\right\|_{0, \gamma}^{2}=\|r\|_{M}^{2}$. We have

$$
\begin{aligned}
\|\mathbf{v}\|_{W}^{2} & =\|\tilde{r}\|_{0, \Omega}^{2}+\left\|r_{f}\right\|_{0, \gamma}^{2}+\|\nabla \varphi\|_{0, \Omega}^{2}+\left\|\nabla_{f} \varphi_{f}\right\|_{0, \gamma}^{2}+2\left\|\mathbf{v}_{\mathbf{1}} \cdot \mathbf{n}_{\mathbf{1}}\right\|_{0, \gamma}^{2} \\
& \leq(1+C(\Omega))\|\tilde{r}\|_{0, \Omega}^{2}+(1+C(\gamma))\|r\|_{0, \gamma}^{2}+C(\Omega)\|\tilde{r}\|_{0, \Omega}^{2} .
\end{aligned}
$$

Remark: The case in which the parameter $\xi=1 / 2$, that appeared to be natural in the derivation of the model, corresponds to a stability limit for this model. In some numerical cases (for instance in the cases presented in Sections 6.1 and 6.2), the model problem gives very good results when the parameter $\xi$ is equal to $1 / 2$. But on some other numerical tests, instabilities appear (see for instance Section 6.3).

Remark: One can prove in the same way that the discrete problem has a unique solution and that it converges towards the continuous solution of the model problem.

\section{Interpretation of the discrete model and error estimate}

We have seen in Section 3 that one could derive the models by simply averaging the transmission problem under its strong formulation (2). In this section, we seek a simple error estimate for the model problem. For this purpose, we show that the discrete model problem for $\xi=1$ and $2 / 3$ is actually none other than the discrete transmission problem under a specific domain decomposition formulation, with certain hypotheses over the approximation spaces and the mesh. Throughout this section, we will assume for simplicity that homogeneous Dirichlet boundary conditions are imposed on $\partial \Omega$.

\subsection{Assumptions concerning the mesh}

Let $\mathcal{T}_{h}=\cup \mathcal{T}_{h, i}$ be a conforming finite element partition of $\bar{\Omega}=\cup \bar{\Omega}_{i}, i=1,2, f$. The meshes $\mathcal{T}_{h, i}, i=$ 1,2 and the mesh $\mathcal{T}_{h, f}$ match on the interfaces $\gamma_{i}, i=1,2$. We will assume henceforth that the following Hypothesis concerning the mesh is true.

Hypothesis 1 We assume that the mesh $\mathcal{T}_{h}$ of the whole domain $\Omega$ posseses the following shape: in the fracture $\Omega_{f}$ there exists only 1 strip of rectangular (2D) or parallelepiped (3D) cells, i.e. in 3D, each cell in the fracture is a parallelepiped with one face on $\gamma_{1}$ and the opposite face contained in $\gamma_{2}$. (See figure (2).)

Of course this hypothesis is quite restrictive, as it forbids in 3D a mesh made exclusively of tetrahedra. However this assumption is made here only to show the link between the discrete transmission problem and the discrete model problem, therefore this restriction is only for theoretical and not computational purposes.

\subsection{Discrete transmission multiblock problem}

Let us define the approximation spaces used in this paper.

$$
\begin{aligned}
& \mathbf{Z}_{\mathbf{i}}=H\left(\operatorname{div} ; \Omega_{i}\right), \quad i=1,2, f . \quad \mathbf{Z}=\bigoplus_{i=1,2, f} \mathbf{Z}_{\mathbf{i}} . \\
& N_{i}=L^{2}\left(\Omega_{i}\right), \quad i=1,2, f . \quad N=\bigoplus_{i=1,2, f} N_{i}=L^{2}(\Omega) .
\end{aligned}
$$

Let

$$
\mathbf{Z}_{\mathbf{h}, \mathbf{i}} \times N_{h, i} \subset \mathbf{Z}_{\mathbf{i}} \times N_{i}, \quad i=1,2, f,
$$



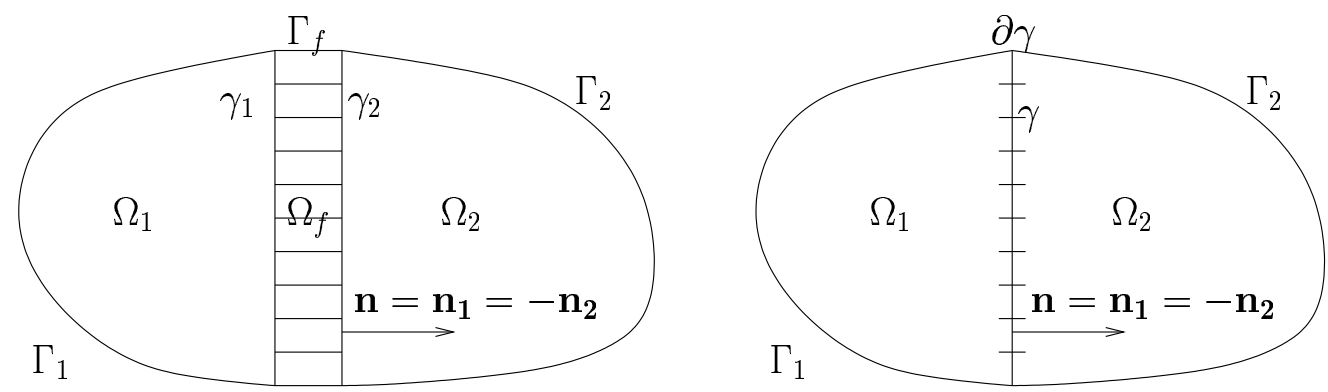

Figure 2: Left: the domain $\Omega$ with a $2 \mathrm{D}$ fracture $\Omega_{f}$ that is meshed with rectangles. Right: the $1 \mathrm{D}$ fracture $\gamma$ is meshed with the projection of the $2 \mathrm{D}$ mesh on $\gamma_{1}\left(\right.$ or $\left.\gamma_{2}\right)$.

be the usual mixed finite element approximation made of Raviart-Thomas (and Nedelec in 3D) spaces of lowest order ([14], [15], [13]). We recall that $Z_{h, i}$ consists of some linear vector functions and that $N_{h, i}$ is made up of cellwise constant scalar functions. Let

$$
\mathbf{Z}_{\mathbf{h}}=\bigoplus_{i=1,2, f} \mathbf{Z}_{\mathbf{h}, \mathbf{i}}, \quad N_{h}=\bigoplus_{i=1,2, f} N_{h, i}
$$

Let

$$
\Lambda_{h}=Q_{0}(\gamma)=\left.\mathbf{Z}_{\mathbf{h}, \mathbf{i}} \cdot \mathbf{n}_{\mathbf{i}}\right|_{\gamma_{i}}=Q_{0}\left(\gamma_{i}\right), \quad i=1,2, f,
$$

be the interface approximation space made up of functions constant on each face. For simplicity, but with an abuse of notation, we use the same notation $\Lambda_{h}$ for the three spaces on the interfaces $\gamma_{1}, \gamma_{2}$ and $\gamma$, as the spaces actually do match.

We write the discrete mixed finite element approximation for the original problem (1) or, equivalently, for the transmission problem (2) introducing interelement multipliers $\left(\lambda_{h, 1}, \lambda_{h, 2}\right)$ (see [8], [4] and references therein). We seek $\mathbf{u}_{\mathbf{h}} \in \mathbf{Z}_{\mathbf{h}}, p_{h} \in N_{h},\left(\lambda_{h, 1}, \lambda_{h, 2}\right) \in \Lambda_{h}^{2}$ such that

$$
\begin{array}{rlrl}
\left(K_{i}^{-1} \mathbf{u}_{\mathbf{h}}, \mathbf{v}\right)_{\Omega_{i}}-\left(\operatorname{div} \mathbf{v}, p_{h}\right)_{\Omega_{i}} & =-\left(\mathbf{v} \cdot \mathbf{n}_{\mathbf{i}}, \lambda_{h, i}\right)_{\gamma_{i}}, \quad \mathbf{v} \in \mathbf{Z}_{\mathbf{h}, \mathbf{i}}, i=1,2, \\
\left(K_{i}^{-1} \mathbf{u}_{\mathbf{h}}, \mathbf{v}\right)_{\Omega_{f}}-\left(\operatorname{div} \mathbf{v}, p_{h}\right)_{\Omega_{f}} & =-\sum_{j=1,2}\left(\mathbf{v} \cdot \mathbf{n}_{\mathbf{f}}, \lambda_{h, j}\right)_{\gamma_{j}}, \quad \mathbf{v} \in \mathbf{Z}_{\mathbf{h}, \mathbf{f}}, \\
\left(\operatorname{div} \mathbf{u}_{\mathbf{h}}, r\right)_{\Omega_{i}} & =\left(q_{i}, r\right)_{\Omega_{i}}, \quad r \in N_{h, i}, i=1,2, f, \\
\left(\mathbf{u}_{\mathbf{h}, \mathbf{1}} \cdot \mathbf{n}_{\mathbf{1}}+\mathbf{u}_{\mathbf{h}, \mathbf{f}} \cdot \mathbf{n}_{\mathbf{f}}, \mu_{h, 1}\right)_{\gamma_{1}} & =0, & \mu_{h, 1} \in \Lambda_{h}, \\
\left(\mathbf{u}_{\mathbf{h}, \mathbf{2}} \cdot \mathbf{n}_{\mathbf{2}}+\mathbf{u}_{\mathbf{h}, \mathbf{f}} \cdot \mathbf{n}_{\mathbf{f}}, \mu_{h, 2}\right)_{\gamma_{2}} & =0, & & \mu_{h, 2} \in \Lambda_{h} .
\end{array}
$$

\subsection{Discrete model problem}

Now we write the discretization of the model problem (28) using the spaces of approximation defined in the previous section. Let

$$
\mathbf{W}_{\mathbf{h}, \gamma} \times \Lambda_{h} \subset H\left(\operatorname{div}_{\tau} ; \gamma\right) \times L^{2}(\gamma)
$$

be the usual Raviart-Thomas mixed finite element space of lowest order defined in the $(n-1)$ dimensional interface $\gamma$. We then define the spaces of approximation of the spaces $\mathbf{W} \times M$ set in Section 4.2. Let

$$
\mathbf{W}_{\mathbf{h}}=\bigoplus_{i=1,2} \mathbf{Z}_{\mathbf{h}, \mathbf{i}} \oplus \mathbf{W}_{\mathbf{h}, \gamma}, \quad M_{h}=\bigoplus_{i=1,2} N_{h, i} \oplus \Lambda_{h} .
$$

The discrete mixed model problem becomes (with homogeneous Dirichlet boundary conditions):

$$
\begin{array}{lll}
\text { Find } \mathbf{u}_{\mathbf{h}}^{\mathbf{m}} \in \mathbf{W}_{\mathbf{h}}, p_{h}^{m} \in M_{h} & \text { such that } & \\
\alpha_{\xi}\left(\mathbf{u}_{\mathbf{h}}^{\mathbf{m}}, \mathbf{v}\right)-\beta\left(\mathbf{v}, p_{h}^{m}\right) & =0 & \forall \mathbf{v} \in \mathbf{W}_{\mathbf{h}} \\
\beta\left(\mathbf{u}_{\mathbf{h}}^{\mathbf{m}}, r\right) & =L_{q}(r) & \forall r \in M_{h} .
\end{array}
$$




\subsection{Link between transmission and model problems}

With these spaces and Hypothesis 1, one can simply eliminate two unknowns of the transmission problem: first the normal component of the Darcy velocity in the fracture can be computed as a function of the Darcy velocities in the neighbouring subdomains from the continuity of the fluxes accross the interfaces $\gamma_{1}$ and $\gamma_{2}$. Second one can eliminate the Lagrange multipliers that enforce the continuity of the pressure on $\gamma_{1}$ and $\gamma_{2}$. This is done with the equation expressing Darcy's law in the fracture written for a test function normal to the fracture. This can be done in two different ways yielding two different parameters $\xi$.

After these eliminations we obtain a new system of equations, the model problem (28). We illustrate this at an algebraic level in Section 5.5.

\subsection{Algebraic system}

The discretization of the transmission problem in a mixed weak form (31) yields the following symmetric system (33). In this section, we do not write the right hand side explicitly, because it is of no interest for our purpose here.

$$
\left[\begin{array}{cccccccc}
A_{1} & B_{1}^{\top} & 0 & 0 & 0 & 0 & E_{1}^{\top} & 0 \\
B_{1} & 0 & 0 & 0 & 0 & 0 & 0 & 0 \\
0 & 0 & A_{2} & B_{2}^{\top} & 0 & 0 & 0 & E_{2}^{\top} \\
0 & 0 & B_{2} & 0 & 0 & 0 & 0 & 0 \\
0 & 0 & 0 & 0 & A_{f} & B_{f}^{\top} & D_{1}^{\top} & D_{2}^{\top} \\
0 & 0 & 0 & 0 & B_{f} & 0 & 0 & 0 \\
E_{1} & 0 & 0 & 0 & D_{1} & 0 & 0 & 0 \\
0 & 0 & E_{2} & 0 & D_{2} & 0 & 0 & 0
\end{array}\right]\left[\begin{array}{c}
U_{1} \\
P_{1} \\
U_{2} \\
P_{2} \\
U_{f} \\
P_{f} \\
T P_{1} \\
T P_{2}
\end{array}\right]=\left[\begin{array}{c}
0 \\
\\
0 \\
0 \\
\\
0 \\
0
\end{array}\right]
$$

Here, $U_{j}, j=1,2, f$ (and $P_{j}, j=1,2, f$ ) represent the velocity (respectively pressure) unknowns in the subdomains $\Omega_{j}, j=1,2, f . T P_{1}$ and $T P_{2}$ are the Lagrange multipliers (trace-of-pressure unknows) living on the interfaces $\gamma_{i}, i=1,2$. The first pair of equations in (33) represents Darcy's law and the conservation equation in $\Omega_{1}$. The matrix $E_{1}^{\top}$ ensures the Dirichlet boundary condition on the interface $\gamma_{1}$. The second pair of equations is the same as the previous pair, but this time in $\Omega_{2}$. The third pair of equations is the same in the domain $\Omega_{f}$, except here there are two matrices $D_{1}^{\top}$ and $D_{2}^{\top}$ for the source term. So far, all these equations enforce continuity of the pressure across the interfaces $\gamma_{1}$ and $\gamma_{2}$ through the Lagrange multipliers $T P_{1}$ and $T P_{2}$. The last two equations respectively enforce continuity of the velocities at the interfaces $\gamma_{1}$ and $\gamma_{2}$.

We decompose the velocities in the fracture $U_{f}$ into a tangential component $U_{f}^{\tau}$ and a normal component $U_{f}^{\mathbf{n}}=\left[U_{f, 1}^{\mathbf{n}} \mid U_{f, 2}^{\mathbf{n}}\right]^{\top}$, which is itself split into a part based on the interface $\gamma_{1}$ and the other on $\gamma_{2}$. (We recall that we have assumed that there is only one strip of cells along the fracture.)

With this notation, the third pair of equations in (33) becomes (34), where the symmetric matrix $A_{f}$ is also split into tangential and normal parts.

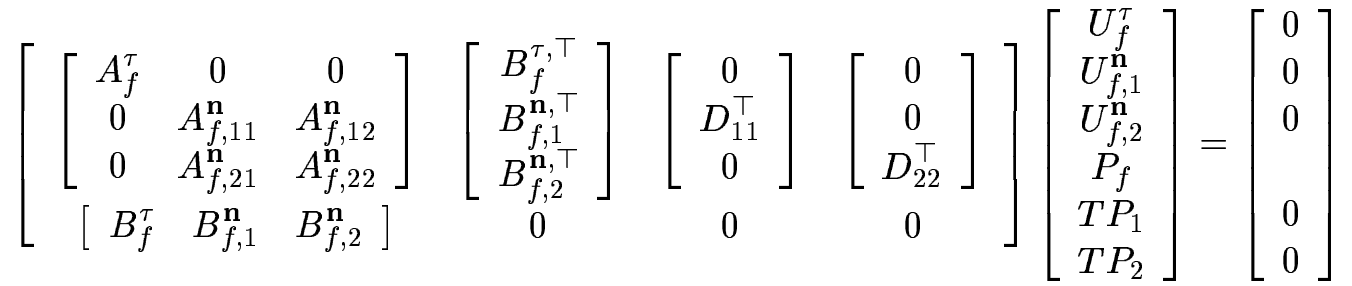

To obtain the discretization of the model problem (22) with $\xi=2 / 3$, it suffices to eliminate the following unknows: the normal velocity in the fracture $U_{n}$ and the two Lagrange multipliers $T P_{1}$ and $T P_{2}$. 
It is easily seen that $D_{11}$ and $D_{22}$ are square and invertible. In the case of $R T_{0}$ spaces, these matrices are actually the identity. So, we first use the last pair of equations in (33) to compute $U_{f, 1}^{\mathbf{n}}$ and $U_{f, 2}^{\mathbf{n}}$ as functions of $U_{1}$ and $U_{2}$.

$$
U_{f, i}^{\mathbf{n}}=D_{i i}^{-1} E_{i} U_{i} \quad i=1,2 .
$$

Plugging $U_{f, i}^{\mathbf{n}}, i=1,2$ into the last equation in (34), we get the conservation equation for the model problem.

$$
B_{f}^{\tau} U_{f}^{\tau}+\sum_{i=1,2} B_{f, i}^{\mathbf{n}} D_{i i}^{-1} E_{i} U_{i}=* .
$$

Next, we eliminate $T P_{i}, i=1,2$, from the second and third equations of (34).

$$
T P_{i}=D_{i i}^{-\top} \sum_{j=1,2} A_{f, i j}^{\mathbf{n}} D_{j j}^{-1} E_{j} U_{j}+D_{i i}^{-\top} B_{f, i}^{\mathbf{n}, \top} P_{f} \quad i=1,2 .
$$

We thus obtain the symmetric system (35). This system corresponds to the discrete model problem (32) when the parameter $\xi=2 / 3$.

$$
\left[\begin{array}{cccccc}
\tilde{A}_{1} & B_{1}^{\top} & \tilde{C}^{\top} & 0 & 0 & \tilde{F}_{1}^{\top} \\
B_{1} & 0 & 0 & 0 & 0 & 0 \\
\tilde{C} & 0 & \tilde{A}_{2} & B_{2}^{\top} & 0 & \tilde{F}_{2}^{\top} \\
0 & 0 & B_{2} & 0 & 0 & 0 \\
0 & 0 & 0 & 0 & A_{f}^{\tau} & B_{f}^{\tau, \top} \\
\tilde{F}_{1} & 0 & \tilde{F}_{2} & 0 & B_{f}^{\tau} & 0
\end{array}\right]\left[\begin{array}{c}
U_{1} \\
P_{1} \\
U_{2} \\
P_{2} \\
U_{f}^{\tau} \\
P_{f}
\end{array}\right]=\left[\begin{array}{c}
0 \\
\\
0 \\
0 \\
\end{array}\right]
$$

with

$$
\tilde{A}_{i}=A_{i}+E_{i}^{\top} A_{f, i i}^{\mathrm{n}} D_{i i}^{-1} E_{i}, \quad \tilde{F}_{i}=B_{f, i}^{\mathrm{n}} D_{i i}^{-1} E_{i} \quad i=1,2,
$$

and

$$
\tilde{C}=E_{2}^{\top} D_{22}^{-\top} A_{f, 21}^{\mathbf{n}} D_{11}^{-1} E_{1}
$$

In this system (35), the terms $\tilde{A}_{i}$ and $\tilde{F}_{i}{ }^{\top}, i=1,2$, ensure a Robin-type boundary condition on the interface $\gamma_{i}, i=1,2$. The unusual coupling term $\tilde{C}$ disapears when using a quadrature rule that kills the extra block-diagonal terms $A_{f, 21}^{\mathbf{n}}=A_{f, 12}^{\mathbf{n}, \top}$ in $A_{f}^{\mathbf{n}}$. This is what happens when the parameter $\xi$ is equal to 1, which amounts to a finite volume modelling (see [7] for instance).

Conclusion: The two systems (35) and (33) are equivalent. The system (35) represents exactly the discretization of model problem (28) when the parameter $\xi=2 / 3$ (or $\xi=1$ with a finite volume discretization).

\subsection{Error estimates}

We have shown that the two algebraic systems (33) and (35) are equivalent, under the hypothesis concerning the spaces of approximation and the mesh. Therefore the error estimates that hold for the transmission problem also hold for the model problem, when the parameter $\xi=2 / 3$.

We obtain the error estimate (36) below. If $\mathcal{T}_{h}$ is a regular family of triangulations of $\Omega$ respecting the assumption (1), there exists a constant $C$ independant of $h$ and $d$, such that if the mixed solution $(\mathbf{u}, p)$ of the original problem (1) is such that $(\mathbf{u}, p) \in\left(H^{1}(\Omega)\right)^{n} \times H^{1}(\Omega)$ and $\operatorname{div} \mathbf{u} \in H^{1}(\Omega)$, and if $\left(\mathbf{u}_{\mathbf{h}}, p_{h}\right)$ in $\mathbf{Z}_{\mathbf{h}} \times N_{h}$ is the solution to the discrete problem (31), we have the standard error estimate

$$
\| \mathbf{u}-\left.\mathbf{u}_{\mathbf{h}}\right|_{\mathcal{H}(\operatorname{div}, \Omega)}+|| p-\left.p_{h}\right|_{L^{2}(\Omega)} \leq C \max \{h, d\}\left(|p|_{1, \Omega}+|\mathbf{u}|_{1, \Omega}+|\operatorname{div} \mathbf{u}|_{1, \Omega}\right) .
$$


We denote by $\left(\mathbf{u}_{\mathbf{h}}^{\mathbf{m}}, p_{h}^{m}\right)=\left(\left(\mathbf{u}_{\mathbf{h}, \mathbf{1}}^{\mathbf{m}}, \mathbf{u}_{\mathbf{h}, \mathbf{2}}^{\mathbf{m}}, \mathbf{u}_{\mathbf{h}, \mathbf{f}}^{\mathbf{m}}\right),\left(p_{h, 1}^{m}, p_{h, 2}^{m}, p_{h, f}^{m}\right)\right)$ the solution to the discrete model problem (32). The solution $\left(\mathbf{u}_{\mathbf{h}}^{\mathbf{m}}, p_{h}^{m}\right)$ that lives in $\mathbf{W}_{\mathbf{h}} \times M_{h}$ is extended to a function $\left(\tilde{\mathbf{u}}_{\mathbf{h}}^{\mathbf{m}}, \tilde{p}_{h}^{m}\right)$ that lives in $\mathbf{Z}_{\mathbf{h}} \times N_{h}$. This is simply done in equation (37). We define the constant extension from $\gamma$ to $\Omega_{f}: E_{f}: L^{2}(\gamma) \rightarrow L^{2}\left(\Omega_{f}\right), E_{f}(p)=p \otimes 1_{[-d / 2, d / 2]}$, where $1_{[-d / 2, d / 2]}$ is the characteristic function of the segment $[-d / 2, d / 2]$.

$$
\begin{array}{ll}
\tilde{\mathbf{u}}_{\mathbf{h}, \mathbf{i}}^{\mathbf{m}}=\mathbf{u}_{\mathbf{h}, \mathbf{i}}^{\mathbf{m}} & \text { in } \Omega_{i}, \quad i=1,2 \\
\tilde{p}_{h, i}^{m}=p_{h, i}^{m} & \text { in } \Omega_{i}, \quad i=1,2 \\
\tilde{\mathbf{u}}_{\mathbf{h}, \mathbf{f}}^{\mathbf{m}}=\left(\left[\left|\mathbf{u}_{\mathbf{h}, \mathbf{i}}^{\mathbf{m}} \cdot \mathbf{n}_{\mathbf{i}}\right|_{\gamma} \mid\right] \frac{x}{d}+\left\{\left.\mathbf{u}_{\mathbf{h}, \mathbf{i}}^{\mathbf{m}} \cdot \mathbf{n}_{\mathbf{i}}\right|_{\gamma}\right\} ; \frac{1}{d} E_{f} \mathbf{u}_{\mathbf{h}, \mathbf{f}}^{\mathbf{m}}\right) & \text { in } \Omega_{f}=\left[-\frac{d}{2}, \frac{d}{2}\right] \times \gamma, \\
\tilde{p}_{h, f}^{m}=E_{f} p_{h, f}^{m} & \text { in } \Omega_{f}=\left[-\frac{d}{2}, \frac{d}{2}\right] \times \gamma .
\end{array}
$$

In equation (37), the expression $\left[\left|\mathbf{u}_{\mathbf{h}, \mathbf{i}}^{\mathbf{m}} \cdot \mathbf{n}_{\mathbf{i}}\right|_{\gamma} \mid\right]$ (respectively $\left\{\left.\mathbf{u}_{\mathbf{h}, \mathbf{i}}^{\mathbf{m}} \cdot \mathbf{n}_{\mathbf{i}}\right|_{\gamma}\right\}$ ) represents the jump of the normal velocity (resp. the mean velocity from $\Omega_{1}$ to $\Omega_{2}$ ) on the interface $\gamma=\partial \Omega_{1} \cap \partial \Omega_{2}$. The extension of the velocity from $\gamma$ to $\Omega_{f}$ is thus composed of a linear approximation in the normal direction (first component along the $O x$ axis) and a constant extension of the tangential component. It is not difficult to see that such an extension lives in $\mathbf{Z}_{\mathbf{h}}$.

Finally we have seen in Section 5.5 that the algebraic solution of the discrete model problem (32) and of the original discrete problem (31) were the same, in the sense that $\left(\mathbf{u}_{\mathbf{h}}, p_{h}\right)=\left(\tilde{\mathbf{u}}_{\mathbf{h}}^{\mathbf{m}}, \tilde{p}_{h}^{m}\right)$. So this yields the error estimate (38) for the discrete model problem.

$$
\left\|\mathbf{u}-\left.\tilde{\mathbf{u}}_{\mathbf{h}}^{\mathbf{m}}\right|_{\mathcal{H}(\operatorname{div}, \Omega)}+\right\| p-\left.\tilde{p}_{h}^{m}\right|_{L^{2}(\Omega)} \leq C \max \{h, d\}\left(|p|_{1, \Omega}+|\mathbf{u}|_{1, \Omega}+|\operatorname{div} \mathbf{u}|_{1, \Omega}\right)
$$

Remark: This error estimate (38) has a limitation: in some cases, the solution of a fractured problem has little regularity. In these cases, the constant in the estimate may become very large and delay the convergence. This is probably what happens in Section 6.3 where the permeability jumps are large.

Remark: This error estimate (38) holds as well when the parameter $\xi=1$ because one gets the same error estimates from a finite volume discretization.

Remark: In the thin subdomain $\Omega_{f}$, the cells are in general very long and narrow. This can produce large errors, but in [12], it was pointed out that the mixed finite element method behaves well even when the cells are thus stretched. Hence the estimates (36) and therefore (38) remain significant despite the thin subdomain $\Omega_{f}$.

\section{$6 \quad$ Numerical results}

Some numerical results are given in this section in order to illustrate the properties of the model presented in this paper. In the first test case (see Section 6.1), we show that the model presented in this paper gives good results under the hypotheses that were made to derive the former model (see [1]): velocity jumps across the fracture that can occur when the tangential permeability is large, are properly modeled. In the second test case (see Section 6.2), one can see that this improved model is able to properly handle a geological barrier (with a small normal permeability in the fracture). It is shown that this was not possible with the former model. The third test case (see Section 6.3) is more difficult: it combines the two previous classes of problems. In the third test case, the fracture has anisotropic permeabilities, thus creating a zone where neither the pressure nor the velocities is continuous across the fracture. These two very stiff test cases show an influence of the parameter $\xi$, and also show that the model can tackle -when the fracture width is small enough- these types of problems quite reasonably. 
The discrete relative $L^{2}$ errors are computed in the following way. A direct $2 \mathrm{D}$ computation is performed with a mixed hybrid method on a fine mesh $\mathcal{T}_{\eta}$ ( $\eta$ being the mesh size, sufficiently small $(\leq 1 / 200))$. This gives a "reference" pressure $P_{\eta}^{\star}$ that we will assume to be a "good" approximation to the solution. We present this pressure for instance in Figure 4, where the grid is considerably coarsened for picture purposes. Note that the grid is locally refined around the fracture. We call $P_{h}^{m}$ the solution computed with the model presented in this paper. We use a square mesh with a mesh size $h(>\eta)$ in each subdomain, and a $1 \mathrm{D}$ mesh in the fracture (with the same mesh size $h$ ). We call $\Pi_{\eta} P_{h}^{m}$ its projection onto the fine mesh $\mathcal{T}_{\eta}$. The square of the error is then equal to

$$
\left\|P_{h}^{m}-P_{\eta}^{\star}\right\|_{L_{h}^{2}(\Omega)}^{2}=\frac{\sum_{C_{\eta} \in \mathcal{T}_{\eta}}\left(\Pi_{\eta} P_{h}^{m}-P_{\eta}^{\star}\right)^{2} \operatorname{meas}\left(C_{\eta}\right)}{\sum_{C_{\eta} \in \mathcal{T}_{\eta}}\left(P_{\eta}^{\star}\right)^{2} \operatorname{meas}\left(C_{\eta}\right)},
$$

where $\mathcal{T}_{\eta}$ is the fine mesh and meas $\left(C_{\eta}\right)$ the measure of the cell $C_{\eta}$.

\subsection{First test-case : large permeability in the fracture and Dirichlet boundary conditions}

The test-case is described in the Figure 3 (left Figure). The lengths of the domain along the $O x$ and $O y$ axes are respectively $L_{x}=2, L_{y}=1$. The permeability tensor in the fracture depends on a parameter $K_{f}$. The permeability in the other subdomains is constant and isotropic: $K=1$. The fracture width is denoted by $d$. Dirichlet conditions hold on the fracture boundaries. Finally, in this test case, the permeability tensors in the fracture are given by

$$
K_{f 1}=K_{f 2}=K_{f} I d,
$$

where $I d$ is the $2 \mathrm{D}$ identity matrix and $K_{f}$ is a parameter greater than 1 . This means that the fluid tends to flow rapidly along the fracture.
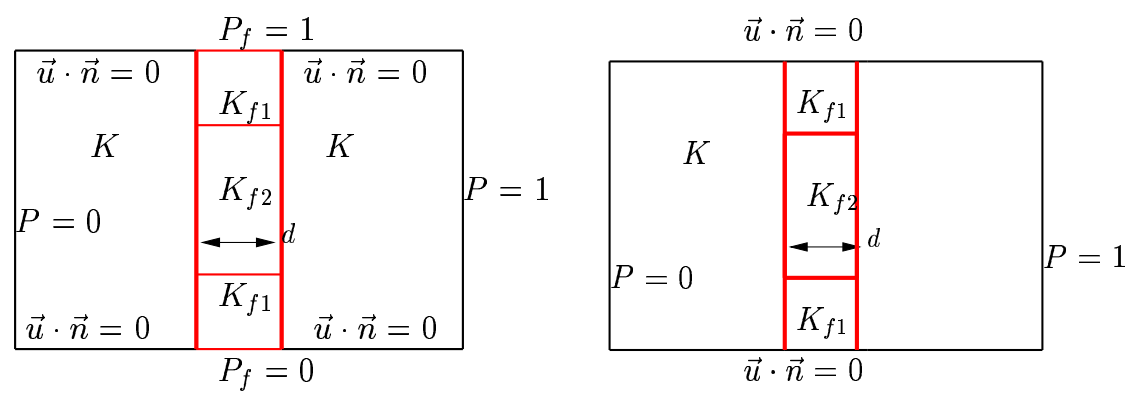

Figure 3: Left: Dirichlet test case with 2 permeability tensors in the fracture. Right: Neumann test case.

One example of a solution given by the model is shown in Figure 5. As a comparison one can see a reference computation performed with a locally refined mesh in Figure 4.

Most of the figures plotting the $L^{2}$ error that follow can be interpreted in three ways: first, for a constant mesh size $h$, we can see the dependence of the model when $d$ tends to 0 ("horizontal" curves). Second, for a constant fracture width $d$ ("vertically"), one can see the convergence of the discrete model solution towards the reference solution. Third, one can see the influence of the parameter $\xi$. The convergence rate was plotted for four values: $\xi=0.51,2 / 3,1,10$. In the Section 6.1 and 6.2 , there is little influence of the parameter $\xi$.

For instance, in Figure 6, (left Figure), the $L^{2}$ errors are plotted as a function of the fracture width $d$, with $K_{f}=1 / d$, for different values of $\xi$ and for different mesh sizes. Here the curves can 
be interpreted in these three ways: first, for a constant $h$, we can hardly see any convergence when $d$ tends to 0. Actually, this is normal as the model very little depends on $d$, but depends on the product $K_{f} d$ that is constant in this test case. Second, for a constant $d$ (small enough: $<1 E-2$ ), when $h$ is divided by a factor 2 , the $L^{2}$ error is divided by a constant factor that is close to 2 . The discrete model solution converges as $\mathcal{O}(\max \{h, d\}$ toward the reference solution: this confirms the previously stated error estimate. The parameter $\xi \leq 1$ has little influence on the solution given by the model in this case. Still, one should not take $\xi$ greater than 1 as in general it degrades the solution.

The behaviour of the solution when the fracture width varies for a fixed fracture permeability is shown in Figure 6, (right Figure). The $L^{2}$ error picture shows again a convergence in $\mathcal{O}(\max \{h, d\})$.
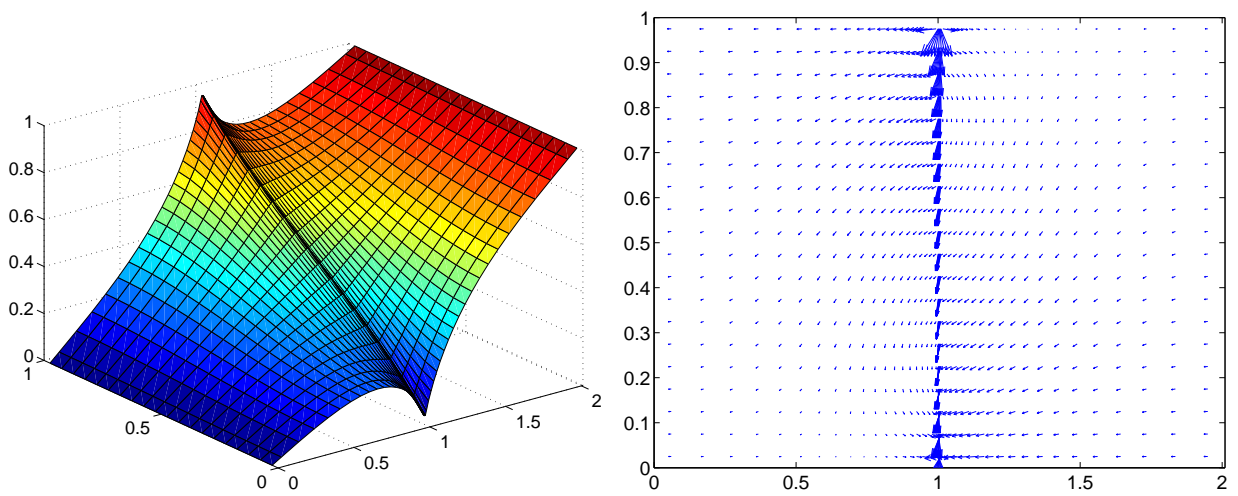

Figure 4: Test case 1. Reference pressure (left) and Darcy velocity (right) given by a locally refined mesh computation. $K_{f}=100, d=0.01$. (The grid is very coarse for picture purposes.)
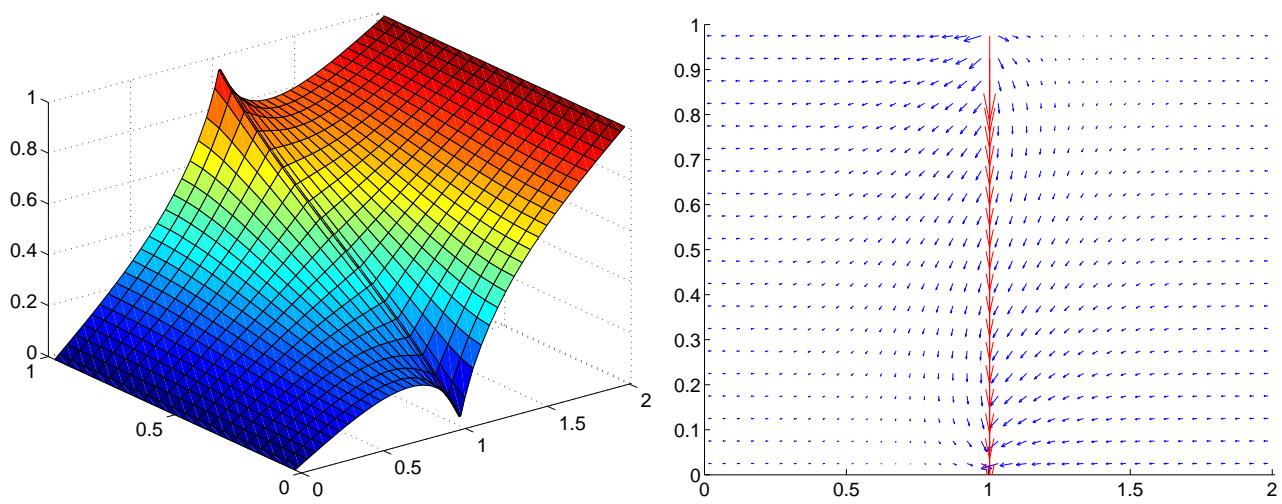

Figure 5: Test case 1. Pressure (left) and Darcy velocity (right) given by the model. $K_{f}=100, d=$ $0.01, \xi=2 / 3$.

\subsection{Second test-case : small permeability in the fracture and Neumann boundary conditions}

The test-case is described in the Figure 3, (right Figure). There is a low permeability fracture in the middle of the domain. Homogeneous Neumann conditions are imposed at the upper part and lower part of the fracture. The permeability tensor in the fracture is given by:

$$
K_{f 1}=I d, \text { and } K_{f 2}=K_{f} I d,
$$

where $I d$ is the $2 \mathrm{D}$ identity matrix and $K_{f}$ is a parameter smaller than 1 . Obviously, the fluid tends to avoid the fracture that represents a geological barrier. 

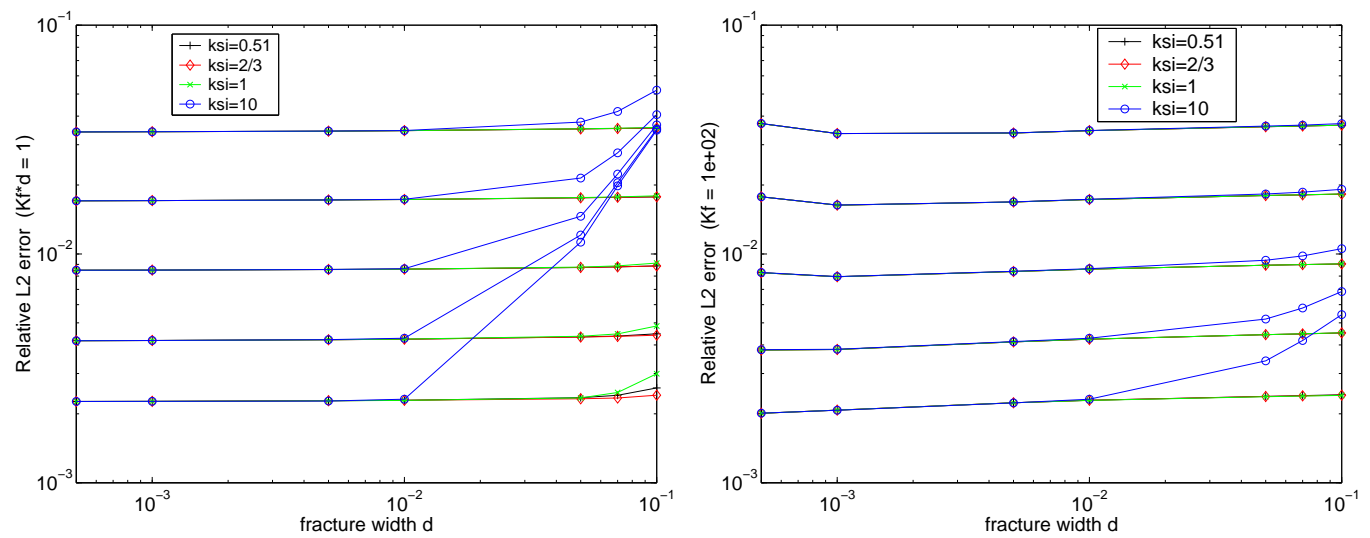

Figure 6: Test case 1. Discrete relative $L^{2}$ error variation as a function of the fracture width $d$, for different values of $\xi$. $\xi=0.51$ in black, $\xi=2 / 3$ in red, $\xi=1$ in green, $\xi=10$ in blue. The five sets of curves show the dependency over the mesh size. From the upper curves to the lower ones: $h=1 / 10$, $h=1 / 20, h=1 / 40, h=1 / 80$, and $h=1 / 160$. Left: the fracture permeability varies as $1 / d$. The permeability increases as the fracture width decreases: $K_{f} \times d=1$. Right: the fracture permeability is constant: $K_{f}=100$.

One example of a solution given by the model is shown in Figure 9. For comparison, a result given by the previous model (with a pressure assumed to be continuous across the fracture, see [1]) is shown in Figure 8. The result given by the previous model is not satisfactory. As a reference, one can see the result of a computation performed with a mesh that is refined around the fracture (see Figure 7).

The behaviour of the solution when the fracture width and the permeability vary in the same manner is shown in Figure 10, (left Figure). There is little dependence of $K_{f} / d$ (which is constant in the model). The behaviour of the solution when the fracture width varies for a fixed fracture permeability is shown in Figure 10, (right Figure).
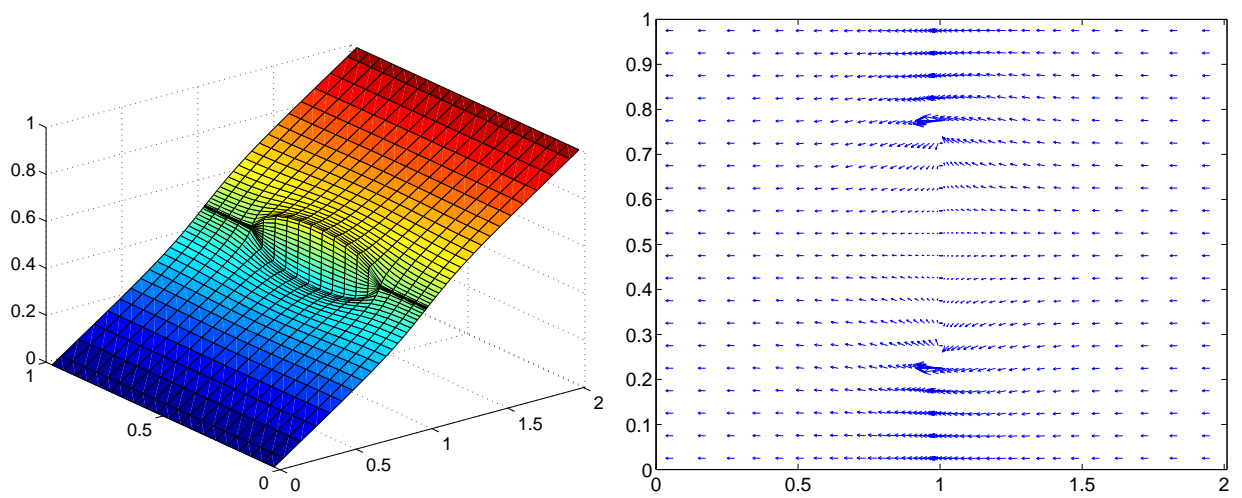

Figure 7: Test case 2. Reference pressure (left) and Darcy velocity (right) given by a locally refined mesh computation. $K_{f}=2 e-3, d=0.01$.

\subsection{Third test-case : 2 anisotropic permeabilities in the fracture and Dirichlet boundary conditions}

This is the same test-case as in Section 6.1 (see the Figure 3, (left Figure)), with a modification in the permeability tensor in the fracture. Dirichlet conditions hold on the fracture boundaries and there are 

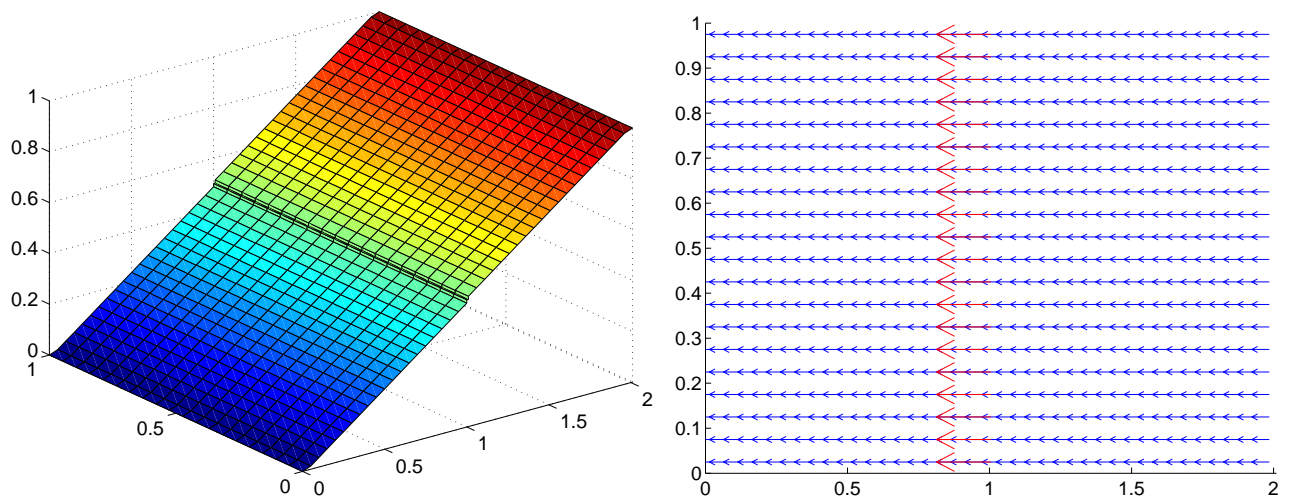

Figure 8: Test case 2. Pressure (left) and Darcy velocity (right) given by the OLD model. $K_{f}=$ $2 e-3, d=0.01$. This result is obviously not satisfactory.
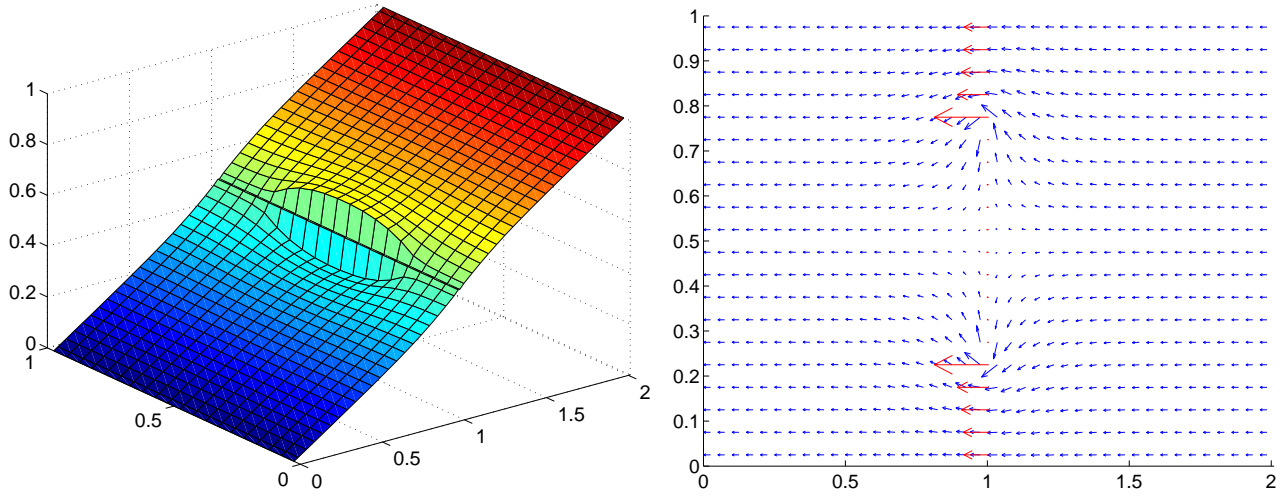

Figure 9: Test case 2. Pressure (left) and Darcy velocity (right) given by the model. $K_{f}=2 e-3, d=$ $0.01, \xi=2 / 3$.
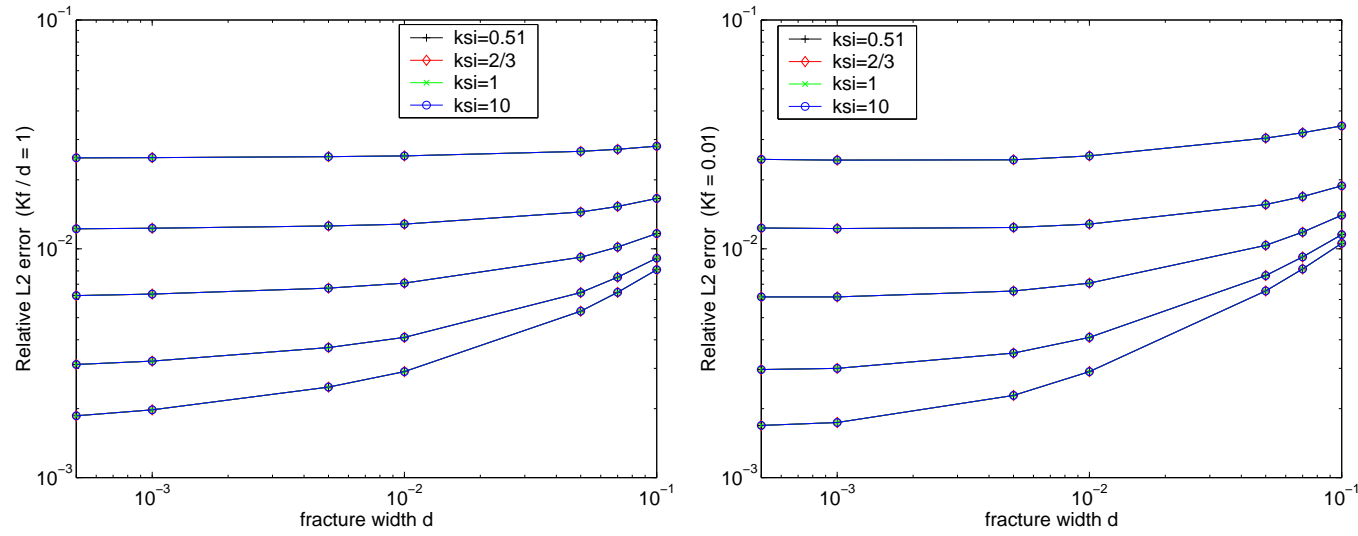

Figure 10: Test case 2. Discrete relative $L^{2}$ error variation as a function of the fracture width $d$, for different values of $\xi$. $\xi=0.51$ in black, $\xi=2 / 3$ in red, $\xi=1$ in green, $\xi=10$ in blue. The five sets of curves show the dependence on the mesh size. From the upper curves to the lower ones: $h=1 / 10, h=1 / 20, h=1 / 40, h=1 / 80$, and $h=1 / 160$. Left: the fracture permeability varies as $d$. The permeability decreases like the fracture width: $K_{f} / d=1$. Right: the fracture permeability is constant: $K_{f}=0.01$. 
2 different anisotropic permeability tensors:

$$
K_{f 1}=\left[\begin{array}{cc}
1 / K_{f} & 0 \\
0 & K_{f}
\end{array}\right] \text { and } K_{f 2}=\left[\begin{array}{cc}
K_{f} & 0 \\
0 & 1 / K_{f}
\end{array}\right]
$$

where $K_{f}$ is a parameter greater than 1 . This means that in the middle part of the fracture where the permeability is equal to $K_{f 2}$, the fluid cannot flow along the fracture, but can easily cross it. It is the contrary in the upper and lower parts of the fracture.

Some examples of solutions given by the model for different parameters $\xi$ are shown in Figures 12, and 13. Some reference results can be seen in Figure (11). One can notice that, on this test case, the model does not approach correctly the reference solution everywhere. This is especially true in the regions close to the fracture, where the permeability tensor is $K_{f 1}$ (extremities of the fracture). It might be due to the high singularities at the exits of the fractures. All the results given by the model strongly depend on the parameter $\xi$, and none of them are completely satisfactory. The lowest errors are generally provided for $\xi=0.51$, but the model with this parameter does not respect the maximum principle: pressure greater than 1 are computed. And for $\xi$ greater than 1 , the $L^{2}$ errors are very large. The best compromise seems to be $\xi=2 / 3$ in this example: the maximum principle is respected and the error remains reasonable.

The behaviour of the solution is shown in Figure 14, when $d$ tends to 0 with a constant product $K_{f} d$. The solution given by the model is almost independent of $K_{f} d$. In Figure 15, the error is plotted as a function of $d$, for two constant values of $K_{f}$. In all these curves, one must note that the error is relatively large, in comparison to the previous test-cases.
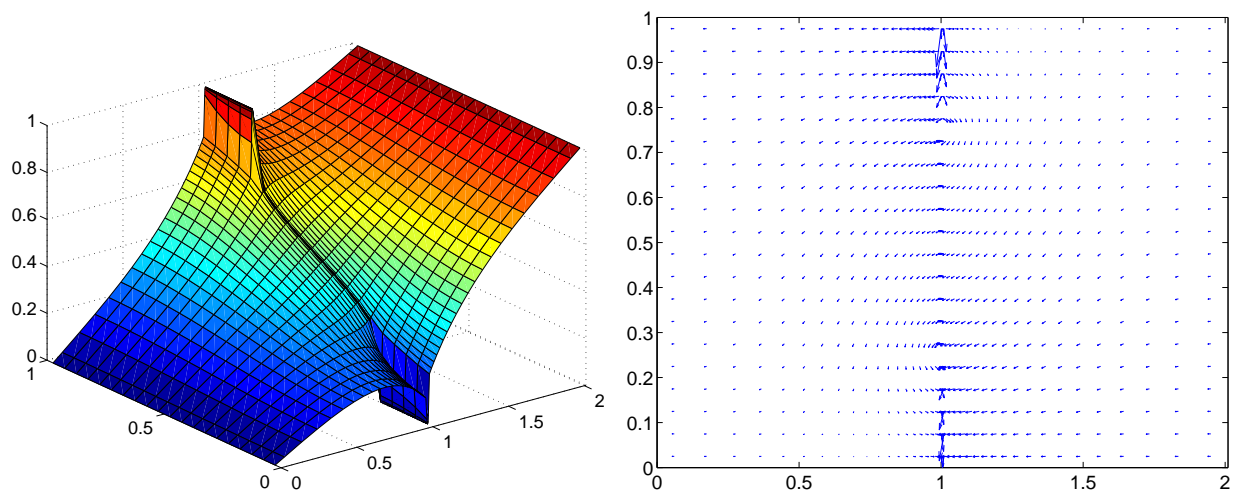

Figure 11: Test case 3. Reference pressure (left) and Darcy velocity (right) given by a locally refined mesh computation. $K_{f}=200, d=0.01$.

\section{Domain decomposition}

In this section we present a way of solving efficiently the problem arising from the model (22).

\subsection{Domain decomposition formulation}

The direct mixed discretization of problem (22) given in equation (35) yields the algebraic system (32). As this system (32) is not positive definite and is very large, it is expensive to solve. The goal is then to eliminate some of the unknowns to obtain a problem easier to solve.

When the parameter $\xi=1$, the model problem reduces to the nonlocal nonstandard positive definite interface problem (44). For other values of the parameter $\xi$, it is not clear yet what to do (see (41)).

Throughout this section, for simplicity, we will assume that homogeneous Dirichlet boundary conditions are imposed on $\partial \Omega$. We introduce the discrete Dirichlet to Neumann operators $S_{1}$ and $S_{2}$ in 

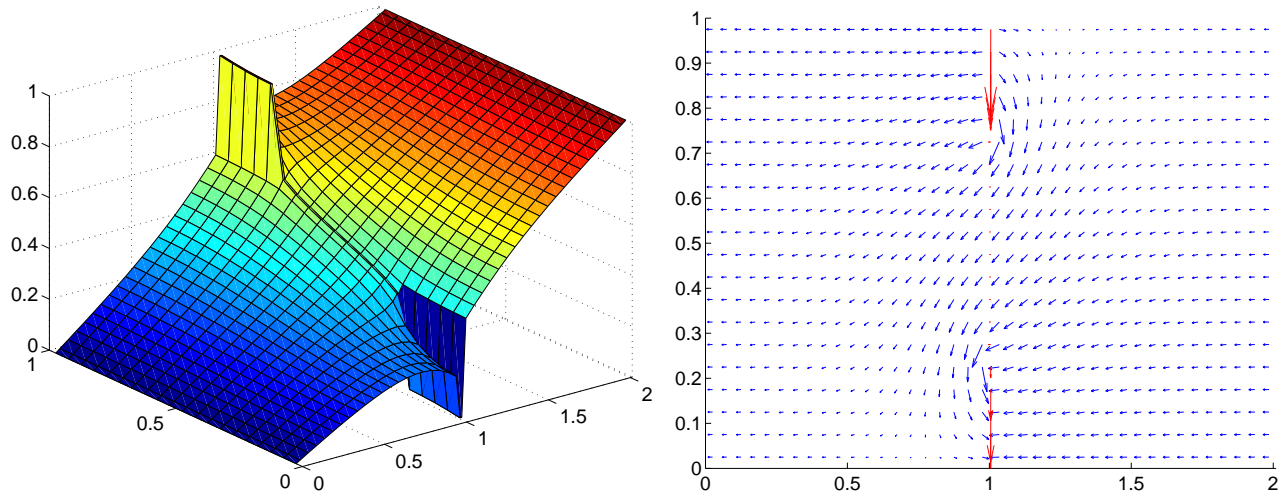

Figure 12: Test case 3. Pressure (left) and Darcy velocity (right) given by the model. $K_{f}=200, d=$ $0.01, \xi=2 / 3$.
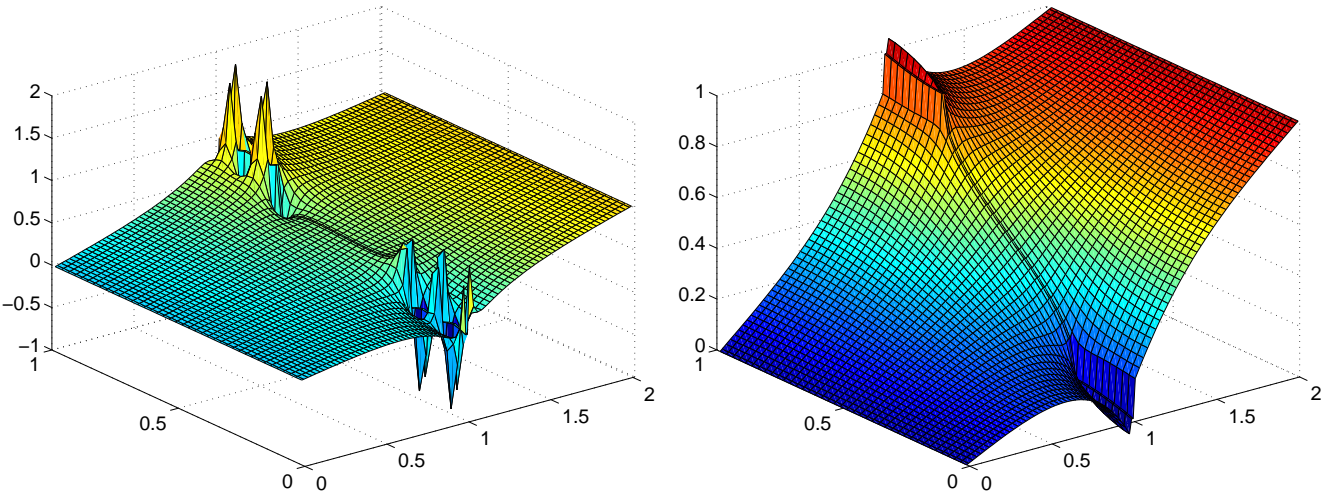

Figure 13: Test case 3. Pressure given by the model. Left: $\xi=0.49$, the parameter $\xi$ is smaller than the stability limit; the model is not stable when $\xi<1 / 2$; scales for the pressure: $[-1,2]$. Right: $\xi=0.51$; small overshoots and undershoots appear at the boundary of the fracture. $K_{f}=200, d=0.01$.

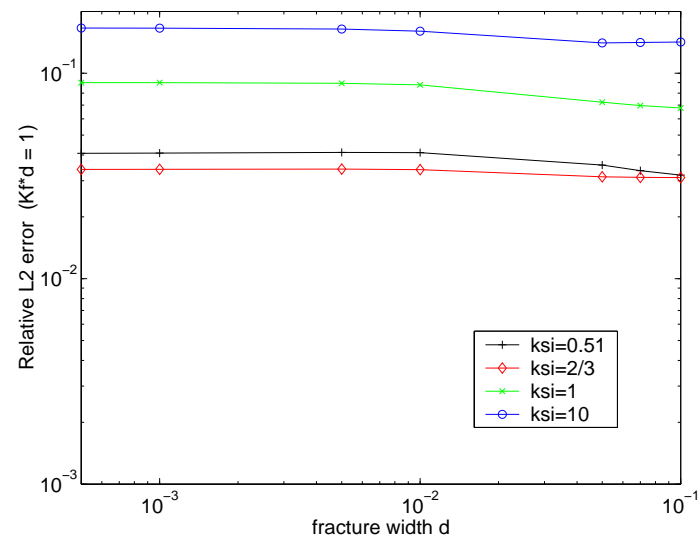

Figure 14: Test case 3. Discrete relative $L^{2}$ error variation as a function of the fracture width, the fracture permeability parameter varying as $1 / d$, for different values of $\xi$. $\xi=0.51$ in black, $\xi=2 / 3$ in red, $\xi=1$ in green, $\xi=10$ in blue. The fracture permeability varies as $1 / d$; the permeability parameter increases as the fracture width decreases: $K_{f} \times d=1 . n x=160, h=6.25 E-3$. Mind the error scales: from $0.1 \%$ up to $20 \%$ : errors remain at a quite high level. 

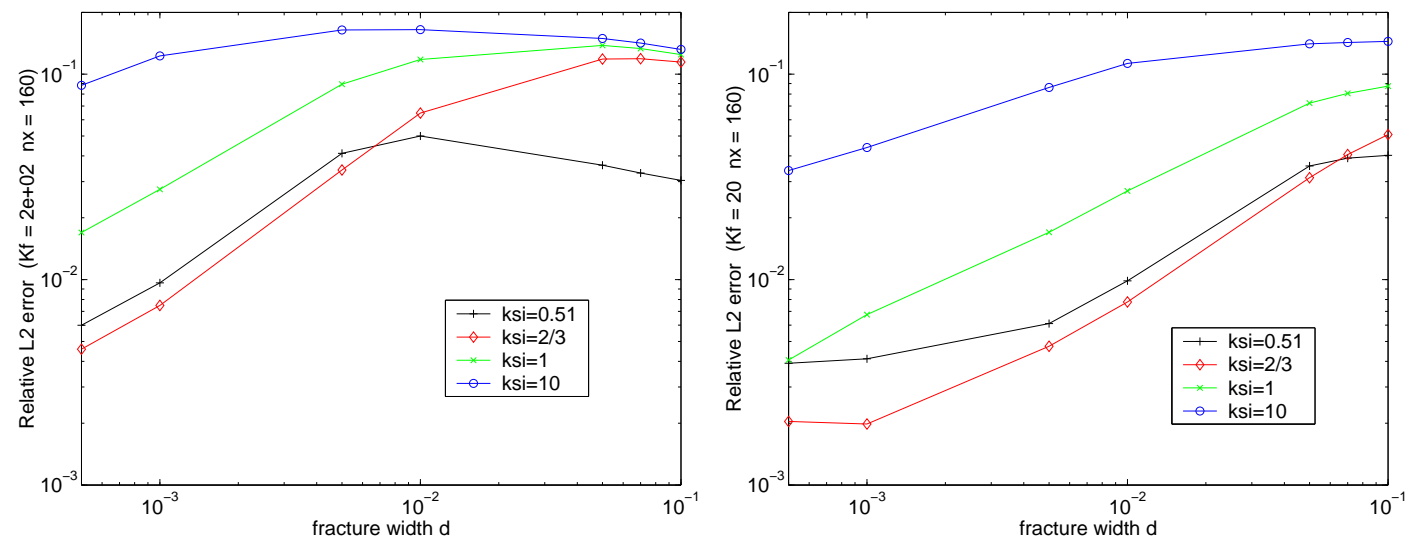

Figure 15: Test case 3. Discrete relative $L^{2}$ error variation as a function of the fracture width, for different values of $\xi$. $\xi=0.51$ in black, $\xi=2 / 3$ in red, $\xi=1$ in green, $\xi=10$ in blue. The fracture permeability parameter is constant. Left: $K_{f}=200$. Right: $K_{f}=20$. For both figures: $n x=160, h=6.25 E-3$. Mind the error scales: from $0.1 \%$ up to $20 \%$ : errors remain at a quite high level.

(39) for the subdomains $\Omega_{1}$ and $\Omega_{2}$ (analogous to the discrete Steklov Poincaré operator, except here they include the source term).

$$
\begin{aligned}
S_{i}: \Lambda_{h} \times N_{h, i} & \rightarrow \Lambda_{h}, \quad i=1,2 \\
S_{i}\left(\lambda_{i}, q_{i}\right) & =-\mathbf{u}_{\mathbf{i}} \cdot \mathbf{n}_{i},
\end{aligned}
$$

so that

$$
\begin{aligned}
\operatorname{div} \mathbf{u}_{\mathbf{i}} & =q_{i} & & \text { in } \Omega_{i}, \\
\mathbf{u}_{\mathbf{i}} & =-\mathbf{K}_{i} \boldsymbol{\nabla} p_{i} & & \text { in } \Omega_{i}, \\
p_{i} & =0 & & \text { on } \Gamma_{i}, \\
p_{i} & =\lambda_{i} & & \text { on } \gamma .
\end{aligned}
$$

In terms of these operators, the problem to be solved, (22), becomes

$$
\begin{aligned}
S_{1}\left(\lambda_{1}, q_{1}\right)+S_{2}\left(\lambda_{2}, q_{2}\right)-\operatorname{div}_{\tau}\left(\mathbf{K}_{f, \tau} d \boldsymbol{\nabla}_{\tau} p_{f}\right) & =q_{f}, \\
\xi S_{1}\left(\lambda_{1}, q_{1}\right)+\alpha_{f} \lambda_{1}-(1-\xi) S_{2}\left(\lambda_{2}, q_{2}\right)-\alpha_{f} p_{f} & =0 \\
-(1-\xi) S_{1}\left(\lambda_{1}, q_{1}\right)+\xi S_{2}\left(\lambda_{2}, q_{2}\right)+\alpha_{f} \lambda_{2}-\alpha_{f} p_{f} & =0
\end{aligned}
$$

and it is not obvious how to solve this problem.

If $\xi=1$, we can express separately $\lambda_{1}$ and $\lambda_{2}$ as functions of $p_{f}$. In this case, we replace the Dirichlet to Neumann operators by the discrete Robin to Neumann operators $\bar{S}_{i}$ defined by (42).

$$
\begin{aligned}
\bar{S}_{i}: \Lambda_{h} \times N_{h, i} & \rightarrow \Lambda_{h}, \quad i=1,2 \\
\bar{S}_{i}\left(\lambda_{i}, q_{i}\right) & =-\mathbf{u}_{\mathbf{i}} \cdot \mathbf{n}_{i},
\end{aligned}
$$

so that

$$
\begin{aligned}
\operatorname{div} \mathbf{u}_{\mathbf{i}} & =q_{i} & & \text { in } \Omega_{i}, \\
\mathbf{u}_{\mathbf{i}} & =-\mathbf{K}_{i} \boldsymbol{\nabla} p_{i} & & \text { in } \Omega_{i}, \\
p_{i} & =0 & & \text { on } \Gamma_{i}, \\
-\mathbf{u}_{\mathbf{i}} \cdot \mathbf{n}_{i}+\alpha_{f} p_{i} & =\alpha_{f} \lambda_{i} & & \text { on } \gamma .
\end{aligned}
$$

Then with $\xi=1$, problem (22) becomes the simpler interface problem (44) that depends on one scalar unknow, $p_{f}$, that lives on the interface.

$$
\bar{S}_{1}\left(p_{f}, q_{1}\right)+\bar{S}_{2}\left(p_{f}, q_{2}\right)-\operatorname{div}_{\tau}\left(\mathbf{K}_{f, \tau} d \nabla_{\tau} p_{f}\right)=q_{f} .
$$


We recall here that the fracture data are present in two different ways in this equation (44). First the product $\mathbf{K}_{f, \tau} d$ plays the role of a mean permeability for the Darcy law equation along the fracture. Also the operators $\bar{S}_{i}, i=1,2$, are Robin to Neumann operators with a specific Robin coefficient that depends on the quotient $\mathbf{K}_{f, \mathbf{n}} / d$.

\subsection{Weak formulation}

It is well known that the Dirichlet to Neumann operators $S_{i}(\cdot, 0), i=1,2$, defined in (39) are symmetric, positive semi-definite (see [8], [4]). We show that this property also holds for the operators defined in (42).

Lemma 1 If the Robin coefficient is positive $\left(\alpha_{f}>0\right)$, the discrete Robin to Neumann operators $\bar{S}_{i}(\cdot, 0)$ are symmetric, positive semi-definite on $\Lambda_{h}$ for $i=1,2$.

Proof: Define the bilinear operators:

$$
\begin{array}{rlrl}
\bar{s}_{i}: \Lambda_{h} \times \Lambda_{h} & \rightarrow \mathbb{R}, & i=1,2, \\
\bar{s}_{i}(\lambda, \mu)=<\bar{S}_{i}(\lambda, 0), \mu> & =-\left(\mu, \mathbf{u}_{\mathbf{h}, \mathbf{i}}(\lambda) \cdot \mathbf{n}_{\mathbf{i}}\right)_{\gamma}, & & i=1,2
\end{array}
$$

with $\mathbf{u}_{\mathbf{h}, \mathbf{i}}(\lambda)$ the solution of the following problem, (46), in $\Omega_{i}, i=1,2$.

Given $\lambda$ in $\Lambda_{h}$, seek $\mathbf{u}_{\mathbf{h}, \mathbf{i}}(\lambda) \in \mathbf{Z}_{\mathbf{h}, \mathbf{i}}$ and $p_{h, i} \in N_{h, i}$, such that for $\mathbf{v} \in \mathbf{Z}_{\mathbf{h}, \mathbf{i}}$ and $r \in N_{h, i}$

$$
\begin{aligned}
\left(K_{i}^{-1} \mathbf{u}_{\mathbf{h}, \mathbf{i}}(\lambda), \mathbf{v}\right)_{\Omega_{i}} & =\left(\operatorname{div} \mathbf{v}, p_{h, i}\right)_{\Omega_{i}}-\left(\mathbf{v} \cdot \mathbf{n}_{\mathbf{i}}, \lambda+\frac{1}{\alpha_{f}} \mathbf{u}_{\mathbf{h}, \mathbf{i}}(\lambda) \cdot \mathbf{n}_{\mathbf{i}}\right)_{\gamma} \\
\left(\operatorname{div} \mathbf{u}_{\mathbf{h}, \mathbf{i}}(\lambda), r\right)_{\Omega_{i}} & =0
\end{aligned}
$$

Take $\mathbf{v}=\mathbf{u}_{\mathbf{h}, \mathbf{i}}(\mu)$ in (46) to see that the operators $\bar{s}_{i}$ can be expressed as

$$
\bar{s}_{i}(\lambda, \mu)=\left(K_{i}^{-1} \mathbf{u}_{\mathbf{h}, \mathbf{i}}(\lambda), \mathbf{u}_{\mathbf{h}, \mathbf{i}}(\mu)\right)_{\Omega_{i}}+\left(\frac{1}{\alpha_{f}^{1 / 2}} \mathbf{u}_{\mathbf{h}, \mathbf{i}}(\lambda) \cdot \mathbf{n}_{\mathbf{i}}, \frac{1}{\alpha_{f}^{1 / 2}} \mathbf{u}_{\mathbf{h}, \mathbf{i}}(\mu) \cdot \mathbf{n}_{\mathbf{i}}\right)_{\gamma}
$$

It is now easy to see that the operators $\bar{s}_{i}, i=1,2$ are symmetric and positive semi-definite.

\subsection{Solving the system efficiently}

In the case $\xi=1$, a good way to solve the model problem (22) is to solve iteratively the linear interface equation (44). To do so, a good idea would be to take a standard finite volume discretization of the Laplace operator $\left(-\operatorname{div}_{\tau}\left(\mathbf{K}_{f, \tau} d \boldsymbol{\nabla}_{\tau} \cdot\right)\right)$ that yields a symmetric, positive semi-definite operator. One has to invert the operator $\bar{S}_{1}(\cdot, 0)+\bar{S}_{2}(\cdot, 0)-\operatorname{div}_{\tau}\left(\mathbf{K}_{f, \tau} d \boldsymbol{\nabla}_{\tau} \cdot\right)$ which is symmetric, positive semidefinite as a sum of such operators (actually it is positive definite because of the Dirichlet boundary conditions). Thus a simple conjugate gradient method can be applied.

This iterative method has been tested numerically and gives the expected results. Some further studies are under way to try to find a preconditioner for this method.

\section{Conclusion}

The models we presented in this paper allow the treatment at the same time and in the same model of the case in which there is a small permeability in the fracture, involving a pressure discontinuity, and the case in which there is a large permeability in the fracture, involving a velocity discontinuity. In each case, the models agree with the asymptotic analysis given by Sanchez-Palencia in [10], [16]. They also give make it possible to treat high anisotropies in the fractures (see Section 6.3), though the 
numerical results are not completely satisfactory and some further studies would probably be useful to understand clearly where the solution lives (as neither the scalar unknown is continuous at the interface, nor its normal derivative).

Some further work is under way to test a more realistic 3-dimensional problem, involving two or three intersecting fractures. These tests should involve also the transport equations. Also a preconditionner for the iterative method that was described in Section 7 will be studied and tested.

\section{Acknowledgement}

We would like to thank Magne Espedal ${ }^{1}$ for useful discussions and remarks.

This work was partially supported by Andra, the French National radioactive waste management agency $^{2}$.

\footnotetext{
${ }^{1}$ Dpt of Math., LIMT, University of Bergen, Norway, Magne.Espedal@mi.uib.no

${ }^{2}$ http://www.andra.fr/
} 


\section{References}

[1] C. Alboin, J. Jaffré, J. E. Roberts, and C. Serres. Domain decomposition for flow in fractured porous media. In C.H. Lai, P. E. Bjorstad, M. Cross, and O. B. Widlund, editors, Domain Decomposition Methods in Sciences and Engineering, pages 365-373. Domain decomposition press, 1999.

[2] Clarisse Alboin, Jérôme Jaffré, Jean E. Roberts, Xuewen Wang, and Christophe Serres. Domain decomposition for some transmission problems in flow in porous media. In Numerical treatment of multiphase flows in porous media (Beijing, 1999), volume 552 of Lecture Notes in Phys., pages 22-34. Springer, Berlin, 2000.

[3] Ph. Angot, T. Gallouët, and R. Herbin. Convergence of finite volume method on general meshes for non smooth solution of elliptic porblems with cracks. In F. Benkhaldoun R. Vilsmeier and D. Hänel Eds, editors, Proc. 2nd Int. Symposium Finite Volumes for Complex Applications, Duisberg, Germany, July 1999, pages 215-222, 1999.

[4] Todd Arbogast, Lawrence C. Cowsar, Mary F. Wheeler, and Ivan Yotov. Mixed finite element methods on nonmatching multiblock grids. SIAM J. Numer. Anal., 37(4):1295-1315 (electronic), 2000 .

[5] Peter Bastian, Zhangxin Chen, Richard E. Ewing, Rainer Helmig, Hartmut Jakobs, and Volker Reichenberger. Numerical simulation of multiphase flow in fractured porous media. In Numerical treatment of multiphase flows in porous media (Beijing, 1999), volume 552 of Lecture Notes in Phys., pages 50-68. Springer, Berlin, 2000.

[6] F. Brezzi and M. Fortin. Mixed and Hybrid Finite Element Methods. Springer-Verlag, Berlin, 1991.

[7] G. Chavent and J. Roberts. A unified physical presentation of mixed, mixed-hybrid finite elements and standard finite difference approximations for the determination of velocities in waterflow problems. Advances in Water Ressources, 14(6):329-348, 1991.

[8] Lawrence C. Cowsar, Jan Mandel, and Mary F. Wheeler. Balancing domain decomposition for mixed finite elements. Math. Comp., 64(211):989-1015, 1995.

[9] I. Faille, E. Flauraud, F. Nataf, S. Pégaz-Fiornet, F. Schneider, and F. Willien. A new fault model in geological basin modelling, application to finite volume scheme and domain decomposition methods. In R. Herbin and D. Kröner Eds, editors, Proc. 3rd Int. Symposium Finite Volumes for Complex Applications, Porquerolles, France, June 2002, pages 543-550, 2002.

[10] Pham Huy Hung and Enrique Sánchez-Palencia. Phénomènes de transmission à travers des couches minces de conductivité élevée. J. Math. Anal. Appl., 47:284-309, 1974.

[11] J. Jaffré, J. Roberts, and V. Martin. Generalized cell-centered finite volume methods for flow in porous media with fault. In R. Herbin and D. Kröner Eds, editors, Proc. 3rd Int. Symposium Finite Volumes for Complex Applications, Porquerolles, France, June 2002, pages 357-364, 2002.

[12] E.F. Kaaschieter. Mixed finite elements for accurate particle tracking in saturated groundwater flow. Advances in Water Resources, 18:227-294, 1998.

[13] J.-C. Nedelec. A new mixed finite elements in $R^{3}$. Numer. Math., 50:57-81, 1986.

[14] P.-A. Raviart and J.-M. Thomas. A mixed finite element method method for second order elliptic problems. In I. Galligani and E. Magenes, editors, Mathematical Aspects of Finite Element Methods; Lecture Notes in Mathematics 606, pages 292-315. Springer-Verlag, Berlin, 1977. 
[15] J.E. Roberts and J.-M. Thomas. Mixed and hybrid methods. In P.G. Ciarlet and J.L. Lions, editors, Handbook of Numerical Analysis Vol.II, pages 523-639. North Holland, Amsterdam, 1991.

[16] Enrique Sánchez-Palencia. Problèmes de perturbations liés aux phénomènes de conduction à travers des couches minces de grande résistivité. J. Math. Pures Appl. (9), 53:251-269, 1974. 


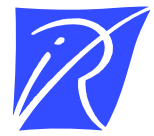

Unité de recherche INRIA Rocquencourt Domaine de Voluceau - Rocquencourt - BP 105 - 78153 Le Chesnay Cedex (France)

Unité de recherche INRIA Lorraine : LORIA, Technopôle de Nancy-Brabois - Campus scientifique 615, rue du Jardin Botanique - BP 101 - 54602 Villers-lès-Nancy Cedex (France)

Unité de recherche INRIA Rennes : IRISA, Campus universitaire de Beaulieu - 35042 Rennes Cedex (France)

Unité de recherche INRIA Rhône-Alpes : 655, avenue de l'Europe - 38330 Montbonnot-St-Martin (France)

Unité de recherche INRIA Sophia Antipolis : 2004, route des Lucioles - BP 93 - 06902 Sophia Antipolis Cedex (France)

INRIA - Domaine de Voluceau - Rocquencourt, BP 105 - 78153 Le Chesnay Cedex (France)

http://www.inria.fr

ISSN 0249-6399 